\title{
Heading in the Right Direction: Understanding Cellular Orientation Responses to Complex Biophysical Environments
}

\author{
Chiara Tamiello, ${ }^{1,2}$ Antonetta B. C. Buskermolen, ${ }^{1,2}$ Frank P. T. Bahijens, ${ }^{1,2}$ Jos L. V. Broers, ${ }^{3}$ \\ and CARLiJn V. C. Bouten ${ }^{1,2}$ \\ ${ }^{1}$ Department of Biomedical Engineering, Eindhoven University of Technology, P.O. Box 513, 5600 MB Eindhoven, The \\ Netherlands; ${ }^{2}$ Institute for Complex Molecular Systems, Eindhoven University of Technology, P.O. Box 513, 5600 \\ MB Eindhoven, The Netherlands; and ${ }^{3}$ Department of Molecular Cell Biology, CARIM School for Cardiovascular Diseases, \\ Maastricht University, P.O. Box 616, 6200 MD Maastricht, The Netherlands
}

(Received 11 August 2015; accepted 10 October 2015; published online 2 November 2015)

Associate Editor Michael R. King oversaw the review of this article.

\begin{abstract}
The aim of cardiovascular regeneration is to mimic the biological and mechanical functioning of tissues. For this it is crucial to recapitulate the in vivo cellular organization, which is the result of controlled cellular orientation. Cellular orientation response stems from the interaction between the cell and its complex biophysical environment. Environmental biophysical cues are continuously detected and transduced to the nucleus through entwined mechanotransduction pathways. Next to the biochemical cascades invoked by the mechanical stimuli, the structural mechanotransduction pathway made of focal adhesions and the actin cytoskeleton can quickly transduce the biophysical signals directly to the nucleus. Observations linking cellular orientation response to biophysical cues have pointed out that the anisotropy and cyclic straining of the substrate influence cellular orientation. Yet, little is known about the mechanisms governing cellular orientation responses in case of cues applied separately and in combination. This review provides the state-of-the-art knowledge on the structural mechanotransduction pathway of adhesive cells, followed by an overview of the current understanding of cellular orientation responses to substrate anisotropy and uniaxial cyclic strain. Finally, we argue that comprehensive understanding of cellular orientation in complex biophysical environments requires systematic approaches based on the dissection of (sub)cellular responses to the individual cues composing the biophysical niche.
\end{abstract}

Keywords-Mechanotransduction, Actin cytoskeleton, Focal adhesion, Strain avoidance, Contact guidance, Structural pathway.

Address correspondence to Carlijn V. C. Bouten, Department of Biomedical Engineering, Eindhoven University of Technology, P.O. Box 513, 5600 MB Eindhoven, The Netherlands. Electronic mail: C.V.C.bouten@tue.nl

Chiara Tamiello and Antonetta B. C. Buskermolen have contributed equally to this work.

\section{INTRODUCTION}

Cardiovascular regenerative medicine has emerged as a promising approach to replace or regenerate damaged or diseased cardiovascular tissues. This interdisciplinary field, at the cross-section of engineering and life sciences, has the potential to restore normal cardiovascular function by using (the properties of) living cells in combination with biomaterials, genes, or drugs. Novel in situ tissue engineering approaches rely on the regenerative potential of the body itself by guiding and controlling cell behavior inside the human body with tailored biomaterials.

The premise of this approach is that, to recapitulate tissue function, an in-depth understanding of native cell behavior under physiological conditions and in response to a biomaterial is needed. Only then, strategies for controlling cell behavior can be designed towards the restoration of tissue functionality and mechanical integrity. ${ }^{52}$

One crucial, but often overlooked, aspect of mimicking native tissue functioning is obtaining and retaining cellular organization. The importance of cellular organization is demonstrated by the fact that biological and mechanical functioning of most tissues is dictated by the cellular arrangement. ${ }^{42}$

The tissues of the cardiovascular system are highly organized. For instance, the myocardial wall, ${ }^{118}$ heart valves $^{120}$ and larger arteries ${ }^{134}$ are characterized by a layered structure with a well-defined cellular arrangement conferring the tissues their native unique anisotropic mechanical behavior needed to perform their function. Given the correlation between structural organization and function, it becomes clear that the loss of cellular organization is indicative of tissue malfunctioning, which can eventually lead to patho- 
physiological conditions. The disorganized arrangement of cardiac cells, for example, is a histological hallmark of cardiac dysfunction in hypertrophic cardiomyopathy. ${ }^{23,58,61,102}$

Cellular organization in cardiovascular tissues depends on the complex interactions between cells, the properties of the microenvironment and the cyclic strains resulting from the hemodynamic environment. Living adherent cells actively interact, respond, and adapt to biochemical and biophysical perturbations. These perturbations trigger intracellular signaling events leading to specific cellular mechanoresponses capable of directing biological relevant processes such as cell differentiation, proliferation and contractility. The mechanisms employed by cells to respond and adapt to the biochemical and biophysical cues of the micro-environment consist of a myriad of distinct but interconnected pathways whose details remain to be unraveled. The outside-in and inside-out feedback loop, referred to as mechanotransduction, is traditionally regarded as the process of converting mechanical stimuli into biochemical signals. Recently, it has been suggested that the structural pathway connecting the extracellular environment to the nucleus ${ }^{149}$ here defined as "the structural mechanotransduction pathway", might be as important as the biochemical transduction pathway for conducting biophysical signal to the nuclear interior. This new concept is supported by the fact that the long-range force propagation into the cell, resulting in deformations deep inside the cytoskeleton and nucleus, occurs 40 times faster than biochemical signaling. ${ }^{97}$ The structural mechanotransduction pathway consists of structural load bearing elements, such as integrins and focal adhesion complexes at the cellular membrane, and actin cytoskeleton stress fibres connected to the nucleus via so-called LINC (Linkers of the Nucleoskeleton and Cytoskeleton) complexes. Experimental evidence for this direct interconnection arises from studies where forces were applied directly to a small spot on the cell surface and consequently induced deformations and movements in the cellular interior. ${ }^{91,93}$ Clearly, defects in the complex and delicate interplay between the cell and its micro-environment resulting, for instance, from aberrations of the structural mechanotransduction pathway, may result in altered cellular mechanoresponse, in case no compensatory signaling mechanisms arise.

The recent development of micro-fabricated devices capable of effectively mimicking controlled biophysical cues has triggered numerous studies aiming at unraveling cellular responses to the properties of the microenvironment. It has become clear that cell orientation is actively determined by the actin stress fibres. ${ }^{132}$ Stress fiber orientation and, consequently, cellular alignment can be induced by two important biophysical cues of the cellular environment, such as those occurring during hemodynamic loading: (1) the anisotropy of the environment, e.g., the substrate on which cells are cultured and (2) uniaxial cyclic strain. ${ }^{7,88}$ These cues induce rapid and specific orientation of the intracellular elements of the structural mechanotransduction pathway, i.e., the focal adhesions, the actin cytoskeleton and the nucleus, suggesting that the direct structural mechanotransduction pathway plays a fundamental role in the cellular orientation response. ${ }^{30,73}$

Although a wealth of information has been obtained by recent in vitro mechanotransduction studies at the tissue-level, single cell observations provide detailed mechanistic insights towards a comprehensive understanding of cellular mechanotransduction. Yet, integrating the results of different investigations is a difficult task because of the complexity of the cellular response, which is not only highly dependent on the choice of the physical and mechanical experimental parameters, but also dependent on the cell-type. Moreover, the effects of combined biophysical cues on the cellular orientation response have just begun to be explored.

Here, we present a state-of-the-art review on the complex interplay between cells, topographical and cyclic strains cues of the extracellular environment, with a focus on cells of the cardiovascular system. Focusing on single cell observations, we first introduce the structural mechanotransduction pathway, i.e., the connected cellular components forming the physical link between the extracellular environment and the nuclear genome. Then, we continue our discussion with a review of experimental observations regarding cellular orientation response to anisotropy of the substrate and uniaxial cyclic strain in two-dimensional (2D) environments. We conclude with a brief outlook on future research directions for improving our current knowledge of cellular mechanoresponse to complex biophysical environments.

\section{THE STRUCTURAL MECHANOTRANSDUCTION PATHWAY: A PHYSICAL CONNECTION BETWEEN THE EXTRACELLULAR ENVIRONMENT AND THE GENOME}

In this section we provide background information on the cellular structural components forming the structural mechanotransduction pathway, i.e., the physical connection between the extracellular matrix (ECM) and the genome contained by the nucleus.

The structural components are represented by the focal adhesions situated at the cell membrane, the cytoskeletal filaments and, at last, the nucleus (Fig. 1). 
Among the cytoskeletal elements we concentrate on the actin filaments, since these structures are directly connected to the focal adhesions and play an important role in determining cell orientation. ${ }^{131,154}$ Moreover their behavior is relatively easy to analyze and quantify from microscopy imaging as they form anisotropic networks when cells are aligned. ${ }^{10,154}$ In this section also the relevance of the nucleo-cytoskeletal connections for correct mechanotransduction is elucidated.

\section{Interconnection Between the Extracellular Environment and the Actin Cytoskeleton}

In vivo adhesive cells are embedded in a filamentous network called extracellular matrix (ECM). The integrins are the first components that physically link the ECM (outside of a cell) with the actin cytoskeleton (inside of the cell). Integrins are transmembrane $\alpha \beta$ heterodimeric receptors that mediate cell adhesion to various ECM ligands such as collagen, fibronectin and laminin. The integrin family consists of about 25 members which are composed of combinations of $\alpha$ and $\beta$ subunits, where the $\alpha$ subunit determines the ligand specificity for cell adhesion to the ECM. ${ }^{68}$ During cell adhesion, conformational changes in the integrins are induced by bidirectional (inside-out and outside-in) signaling of mechanical and biochemical signals across the cell membrane., ${ }^{4,113,114}$ Ligand binding to the integrins leads to clustering of integrin molecules at the cell membrane and recruitment of actin filaments inside the cell. The result of this process is the formation of the so-called nascent focal adhesion complexes (Fig. 1a, left inset), multi-molecular complexes that consist of a large number of different proteins, including talin, vinculin, paxillin and tensin.

Focal adhesion complex formation initially starts with immature, small structures (approximately $100 \mathrm{~nm}$ in diameter ${ }^{45}$ ). These structures reside at the leading edge in protrusions of the cells and provide the structural links between the ECM and the actin cytoskeleton. Strikingly, the maturation of the small focal adhesion complexes into bigger, mature focal adhesions is dependent on actin cytoskeleton bundling and generation of mechanical force. The actin cytoskeleton spans the whole cytoplasm of eukaryotic cells, continuously remodels and reorganizes to perform specific cellular functions. ${ }^{95,140}$ It is made of globular actin (G-actin), which continuously polymerizes into semi-flexible actin filaments, the filamentous actin (F-actin). F-actin assembles into bundles of fibres interconnected by actin crosslinkers (such as alpha-actinin and filamin) and motor proteins such as myosin II. ${ }^{103}$ These bundles of F-actin fibers are referred to as stress fibers. The presence of myosin II within the stress fibers is responsible for their contractility. The newly formed focal adhesions (FAs) reside in both central and peripheral regions of the cell. During this process the morphology of the FAs changes from a dot-like structure to a bigger and more elongated structure $(2-10 \mu \mathrm{m}){ }^{22,44}$ This happens also as a consequence of the recruitment at the adhesion complex of several other proteins, for instance zyxin and alpha-actinin. ${ }^{163} \mathrm{~A}$ critical molecule for both maturation of FAs and mechanosensing is focal adhesion kinase (FAK). This molecule is involved in the transmission of external signals to the cytoskeleton by phosphorylation. ${ }^{50}$

The maturation of FAs provides stable adhesive interconnections between the stress fibers and the ECM. This allows the cell to probe its complex biophysical environment in various directions and over large temporal and spatial scales. ${ }^{121}$ Focal adhesions do not actively generate forces, but rather serve to regulate force transmission between the cytoskeleton and ECM. ${ }^{104}$ The actin cytoskeleton is the intracellular structure able to impose increasing forces when facing growing resistance. This confers the actin cytoskeleton intrinsic mechanosensing and ability to adapt to developing mechanical cues of the cellular environment. However, to which extent stress fibers participate in sensing and transducing environmental signals has not been fully elucidated yet.

\section{Diseases Associated with Dysfunctional ECM-Actin Connections}

The relevance of correct functioning of all components within the mechanotransduction route between ECM and the actin cytoskeleton has become clear in numerous studies in the past few years.

Starting at the ECM, its composition appears to have major impact on cellular behavior. Either weakening or increased ECM stiffness due to decreased or increased amount of collagen evokes a cellular response, which, upon disturbed mechanotransduction, can lead to an even more deregulated ECM. For example, cardiac tissue damage that normally causes controlled levels of enhanced myofibroblast proliferation and collagen production, will, without proper feedback due to disturbed mechanosensing, lead to cardiac fibrosis and stiffening of the cardiac muscle. ${ }^{92}$ For a recent review on the interplay between ECM and mechanotransduction, see Ref. 43

Not only amounts of ECM components but also abnormalities in the molecular composition of its components lead to disturbed mechanosignaling. For instance, mutations in the ECM protein fibrillin-1 as seen in the Marfan syndrome, cause the development of cardiomyopathies in affected mice due to disturbed 


\section{(a)}

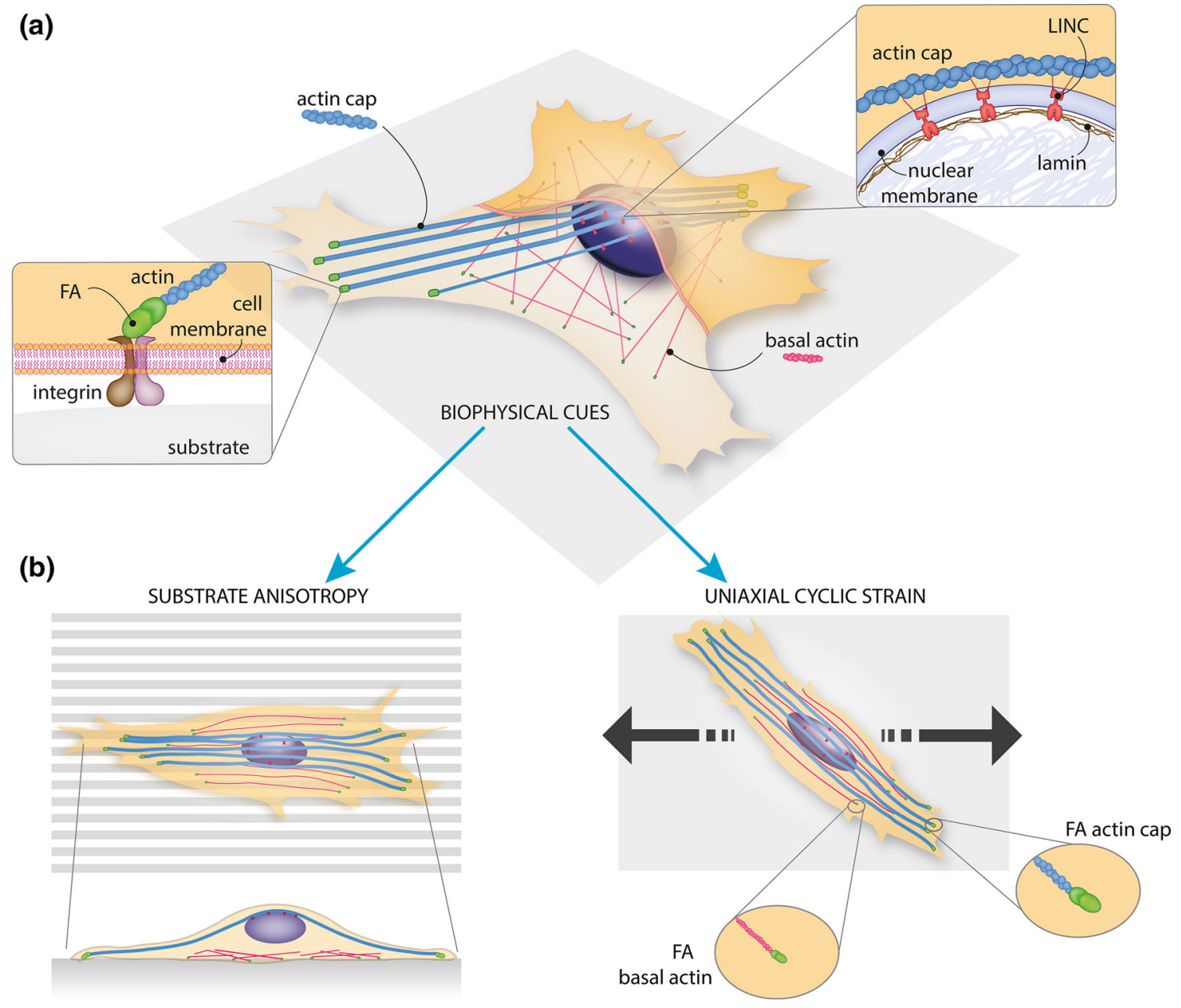

FIGURE 1. The structural mechanotransduction pathway and cellular orientation response to anisotropy of the substrate and uniaxial cyclic strain. (a) Schematic illustration highlighting the (protein) structural elements forming the structural mechanotransduction pathway. Integrins at the plasma membrane connect the extracellular environment (substrate) to the actin cytoskeleton. The connection is realized, in the cellular interior, by the focal adhesion complex. Within the actin cytoskeleton filaments, two kinds of fibers can be distinguished. The basal actin fibers (pink) that can be found underneath the nucleus and the actin cap fibers running on top of the nucleus (cyan). Actin cap fibers are connected to the nuclear interior via the LINC complex and lamins, a group of proteins underlying the nuclear membrane. This network of components forms a direct connection between the extracellular environment and the nuclear interior and function as a fast passing system for the biophysical stimuli. (b) Schematic illustration of cellular response to substrate anisotropy and uniaxial cyclic strain. When plated on an anisotropic substrate (left), the cell tends to align in the direction of the anisotropy. Focal adhesions as well as the actin cytoskeleton align accordingly. The side view shows the arrangements of the actin cap and basal actin fibers. Upon uniaxial cyclic strain (right), the cell responds by strain avoidance. The focal adhesions and the actin cytoskeleton align at an angle with respect to the straining direction. Overall cell orientation coincides with the actin cytoskeleton orientation. Note that the focal adhesions associated with the actin cap fibers are bigger than those associated with the basal actin fibers. Figure by Anthal Smits.

mechanosignaling. Normalizing the extracellular matrix composition in these mice resulted in disappearance of these symptoms. ${ }^{25}$

Proteins, assembled in the FA complex, including the transmembrane integrins as well as the other binding partners, mostly localized at the cytoplasmic side of the cellular membrane, all appear to be critical for the transmission of extracellular forces. Especially, any structural abnormalities in integrins have a devastating effect on mechanotransduction. Even minor modifications of the $\beta 1$-integrin gene resulted in multiple defects in mechanotransduction signaling in adult cardiomyocytes. ${ }^{86}$ An overwhelming number of diseases now have been assigned to failure in any of the other components of the FAs, based on mouse models. ${ }^{90}$ While not all of these diseases are caused by disturbed mechanotransduction, it has become clear that especially improper functioning of the key enzyme FAK leads to severe abnormalities in heart development and heart failure. ${ }^{117}$

While this brief overview suggests that most mechanotransduction diseases are associated with heart (muscle) tissue there is growing evidence that 
impaired or sustained mechanotransduction at the cellular boundaries leads to several other diseases. Abnormal mechanical stimulation can switch on signaling pathways such as beta-catenin signaling in adenomatous polyposis coli (APC) deficient colon tissue, stimulating the development of colon cancer. ${ }^{156}$ Moreover ECM stiffness can drive epithelial to mesenchymal transition of cancer cells, ${ }^{153}$ increasing the malignant behavior of these cells.

\section{Interconnection Between the Actin Cytoskeleton and the Nucleus}

In the surrounding of the nucleus, a subset of actin stress fibers have been found to organize in thick parallel and well-ordered bundles of fibers, physically anchored to the apical surface of the nucleus. ${ }^{75,76}$ Wirtz and coworkers have made an effort to characterize these fibers (actin cap) which are strikingly terminated by wide, long and dynamic focal adhesions (Fig. 1a). ${ }^{17,64,75}$ First, they have demonstrated that the actin cap stress fibers differ from the conventional stress fibers found below the nucleus (basal actin layer, Fig. 1b). By containing more myosin II and the actin bounding protein alphaactinin, actin cap stress fibers are very contractile and highly dynamic. ${ }^{98}$ Furthermore, these fibers not only play a major role in shaping and positioning the nucleus, ${ }^{19,64,75,77,98,139}$ but they are also involved in mechanosensing of substrate elasticity. For instance, cells without an actin cap were observed to be less responsive to changes in matrix elasticity. Finally, fast mechanotransduction also seems to be enabled by this subset of stress fibers. In their study, Chambliss et al. showed that, in response to shear stress stimulation, cells without the actin cap build up thick stress fibers in a shorter time span as compared to in response to biochemical stimulation. ${ }^{18}$ From these findings it has become clear that the perinuclear actin cap is a key component of the physical pathway from the ECM to the nuclear interior for mechanosensing and mechanotransduction.

The coupling between the perinuclear actin cap and the nucleus (nucleo-cytoskeletal connection) is mediated by a group of recently discovered proteins, referred to as the LINC complex (Linker of Nucleoskeleton and Cytoskeleton, Fig. 1a right inset). ${ }^{26,108,127}$ Hooking at the cytoplasmic side of the nucleus, on the outer nuclear membrane (ONM), we find the nesprins (KASH domains proteins), which are connected to the various cytoskeletal filaments. ${ }^{125,166}$ Among the four variants of nesprins, nesprin-1 and -2 bind to actin filaments. ${ }^{54}$ Nesprins, in turn, bind to SUN domain proteins spanning the whole nuclear envelope reaching the nuclear interior. SUN proteins then bind to lamins, a family of type $\mathrm{V}$ intermediate filaments underlying the inner nuclear membrane
(INM). ${ }^{56}$ Lamins in turn physically connect to chromatin. Thus, in this way a physical bridge is formed from the cellular exterior via focal adhesion complexes, actin, the LINC complex, and lamins to chromatin.

Lamins form an elastic meshwork called nuclear lamina (Fig. 1a, right inset). ${ }^{29,109}$ Lamins consist of two main subtypes, A- and B-type lamins (encoded by the gene LMNA, or LMNB1 and LMNB2 respectively). ${ }^{53}$ While B-type lamins are essential for cell survival, A-type lamins are thought to contribute significantly to the maintenance of mechanical integrity of the nucleus. ${ }^{12,63,80,138}$ The nuclear lamina interacts also with the chromatin of the nucleoplasm, and therefore plays a major role in gene expression, DNA replication and repair, chromatin organization and transcriptional response. ${ }^{35,49,122,168}$

The role of the nucleo-cytoskeletal connection in force transmission has been examined recently by many groups. The results of various experimental approaches based on two- and three-dimensional substrates or application of mechanical load, have shown that the structural integrity of this connection is indeed needed for propagation of forces to the nucleus. Indirect demonstration has come from studies employing LMNA-depleted cells. By using this model, it has been shown that nuclear deformations in response to local cellular membrane stretch are completely abolished..$^{91}$ In addition, the studies by Poh et al. ${ }^{111}$ and Zweger et al. ${ }^{169}$ have provided direct evidence that forces are not transmitted to the nucleus when LMNA is depleted from cells, thus when the nucleo-cytoskeletal connection is lost. Recently, it has emerged as well that the tension exerted by the actin on the nucleus directly mediates the spatial polarization of nuclear lamina and the intranuclear architecture. ${ }^{78} \mathrm{In}$ cells lacking A-type lamins, the formation of a nuclear actin cap is partially abolished. ${ }^{75}$ Also, the impaired activation of mechanosensitive genes has been reported in studies with cells lacking A-type lamins. ${ }^{62,81}$

A number of other studies in which either the LINC complex was disrupted or a loss of lamins was induced, support these findings adding that also other cellular functions such as migration, polarization and developmental processes become affected. ${ }^{14,83}$

Although the role of the LINC complex in force propagation to the nucleus has been clarified, controversy remains about its impact on the activation of mechanotransduction pathways. Clues to understand these mechanisms might come from studying diseases arsing form mutations in any of the components connecting actin to the nucleus.

\section{Diseases Associated with Defective Actin-Nucleus Coupling}

Mutations in the LMNA gene encoding for A-type lamins in the nuclear lamina cause a broad spectrum of 
genetic diseases, collectively referred to as laminopathies. ${ }^{13}$ Several hundred mutations in the gene have been discovered and most of them have tissue-specific phenotypes. Twelve different diseases are included into this group: those affecting striated muscle (ranging from Emery/Dreifuss muscular dystrophy (EDMD) to dilated cardiomiopathy with conduction system defects (DCM-CD) and Limb-girdle muscular dystrophy (LGDM), those affecting the adipose tissue (partial lipodystrophy of Dunningan type (FPLD) and those affecting the nervous system (Autosomal recessive Charcot-Marie-Tooth type 2 and Autosomal dominant axonal Charcot-Marie-Tooth disease). However, primary laminopathies can also affect tissues in a systemic fashion and cause premature-aging syndromes like Restrictive Dermopathy (RD) and Hutchinson-Gilford progeria syndrome (HGPS). ${ }^{159}$ The mechanisms underlying tissue-specific effects observed in laminopathies are still largely unknown. Especially in the most diffuse laminopathies, the muscular dystrophies and cardiomyopathies, ${ }^{16}$ it might well be that the lack of structural integrity, thus the susceptibility to mechanical stress could result in altered chromatin organization which, on its turn, results in altered gene expression.

Recently it was observed that also mutations in other components of the LINC complex (e.g., emerin, nesprin-1, nesprin-2, etc.) can give rise to the same disease pathology as seen in EDMD due to LMNA mutations. ${ }^{8,87,94,164}$ Next to this, combinations of mutations in the nucleo-cytoskeletal system have been shown to lead to more severe diseases than the individual component mutations..$^{85,96,129}$ At the same time, it has been suggested that the biochemical signals coming from the cytoplasm might take over or compensate for the lack of the physical nucleo-cytoskeletal interconnection. ${ }^{14,91}$

Altogether, the examples above demonstrate that several structural components of the mechanotransduction pathway connecting the cellular micro-environment and the nuclear interior have been identified. While we do not know the degree of completeness of our understanding, we can confidently state that the structural interconnection is crucial for determining the cellular mechanoresponse.

\section{CELLULAR ORIENTATION RESPONSE TO SUBSTRATE ANISOTROPY AND CYCLIC STRAIN}

In the previous section, in order to appreciate the inside-in part of the cellular mechanotransduction, i.e., how environmental signals are transmitted to the nucleus, we introduced the components of the structural mechanotransduction pathway interconnecting the extracellular environment and the nucleus. To get a comprehensive understanding of the interplay between cellular responses and complex biophysical environments, it is also necessary to have a deep understanding of the inside-out signaling used for cellular mechanoresponse, i.e., how cells respond to environmental cues and which mechanisms are employed by cells for mechanoresponse. In this section we discuss the cellular orientation response to substrate anisotropy and uniaxial cyclic strain (Fig. 1b), focussing on the main components of the structural mechanotransduction pathway, i.e., the focal adhesions, the actin cytoskeleton and the nucleus.

\section{Cellular Orientation Response to Substrate Anisotropy}

Various biophysical cues such as topography, cyclic strain and the mechanical properties of the extracellular environment can induce the alignment of adherent cells by promoting an anisotropic arrangement of structural components at the subcellular level. In 1912, Harrison reported for the first time that the topography of a substrate could influence cell behavior. ${ }^{57}$ Weiss confirmed this in 1945, with the observation that cells preferentially orient and migrate along fibers, an organization principle he named contact guidance. ${ }^{155}$ Today the connotation of this term is slightly different. Contact guidance is now regarded as the ability of cells to sense and align with the anisotropy of the surrounding micro-environment.

Recent developments in microfabrication technologies have led to the manufacturing and application of a variety of substrates with different geometries and length scales, from which several substrates can be used to study contact guidance. Observations obtained using microfabricated substrates engineered to induce contact guidance, have confirmed that a variety of tissue cells, ranging from endothelial cells, ${ }^{38,135,136}$ to fibroblasts, ${ }^{37,39,105,141,142}$ and smooth muscle cells ${ }^{116}$ orients along the direction of the anisotropy of the substrate. A summary of illustrative studies showing the response of cells of the cardiovascular system to anisotropic features of the culture substrate in the submicrometer to micrometer scale is reported in Table 1.

The most used substrates for studying contact guidance are microgrooves, i.e., microengineered arrays of parallel micrometer-sized grooves and ridges. When culturing adherent cells on these substrates it is observed that, at the subcellular level, the focal adhesions and actin fibers follow cellular orientation (Fig. 1b, left). However, the specific response of these structural cellular components depends on many parameters such as groove width, ${ }^{38,39,119,136,142}$ ridge width, ${ }^{11,37-39,130,136}$ groove height ${ }^{11,24,130,135,142}$ and 


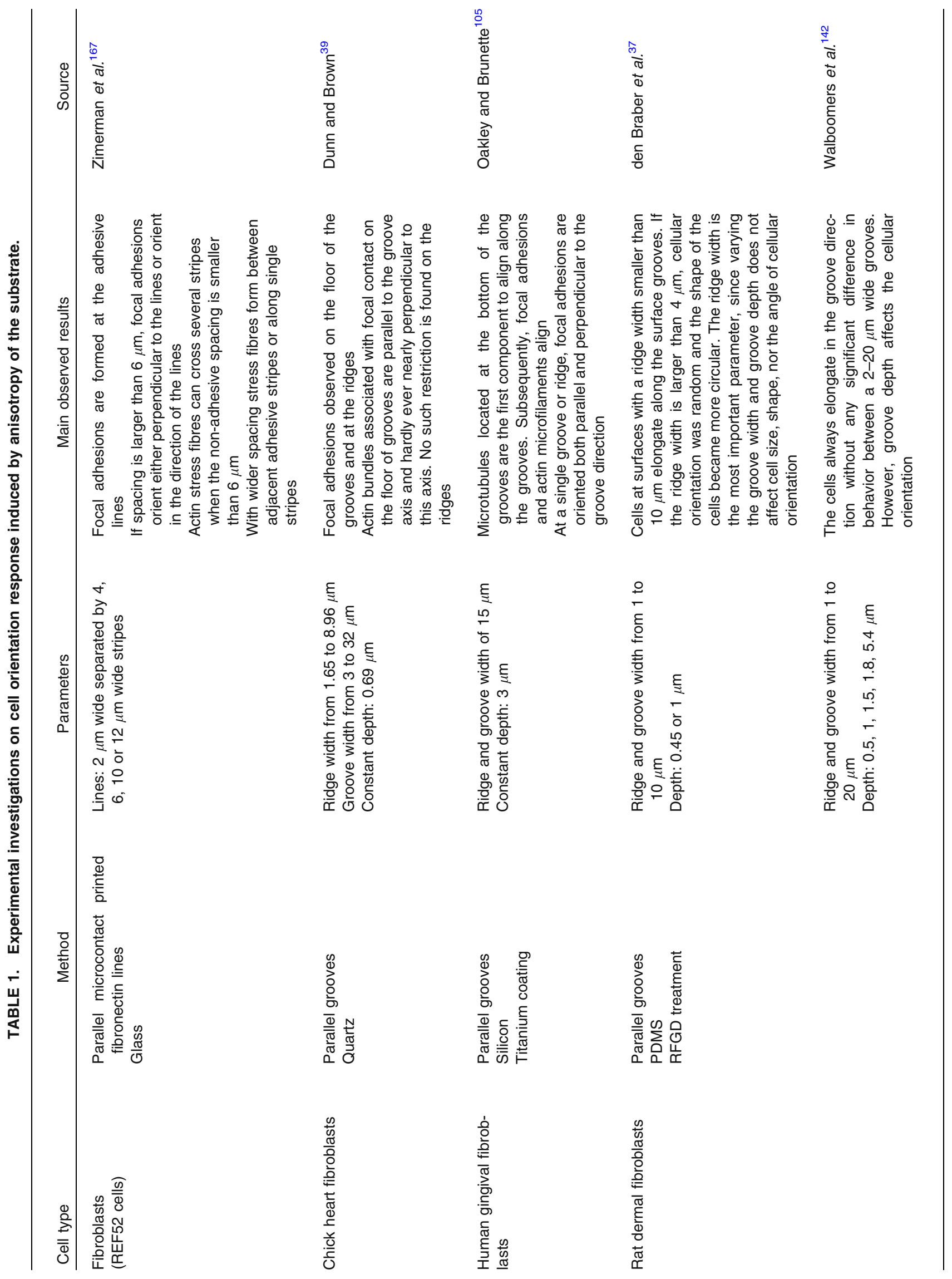




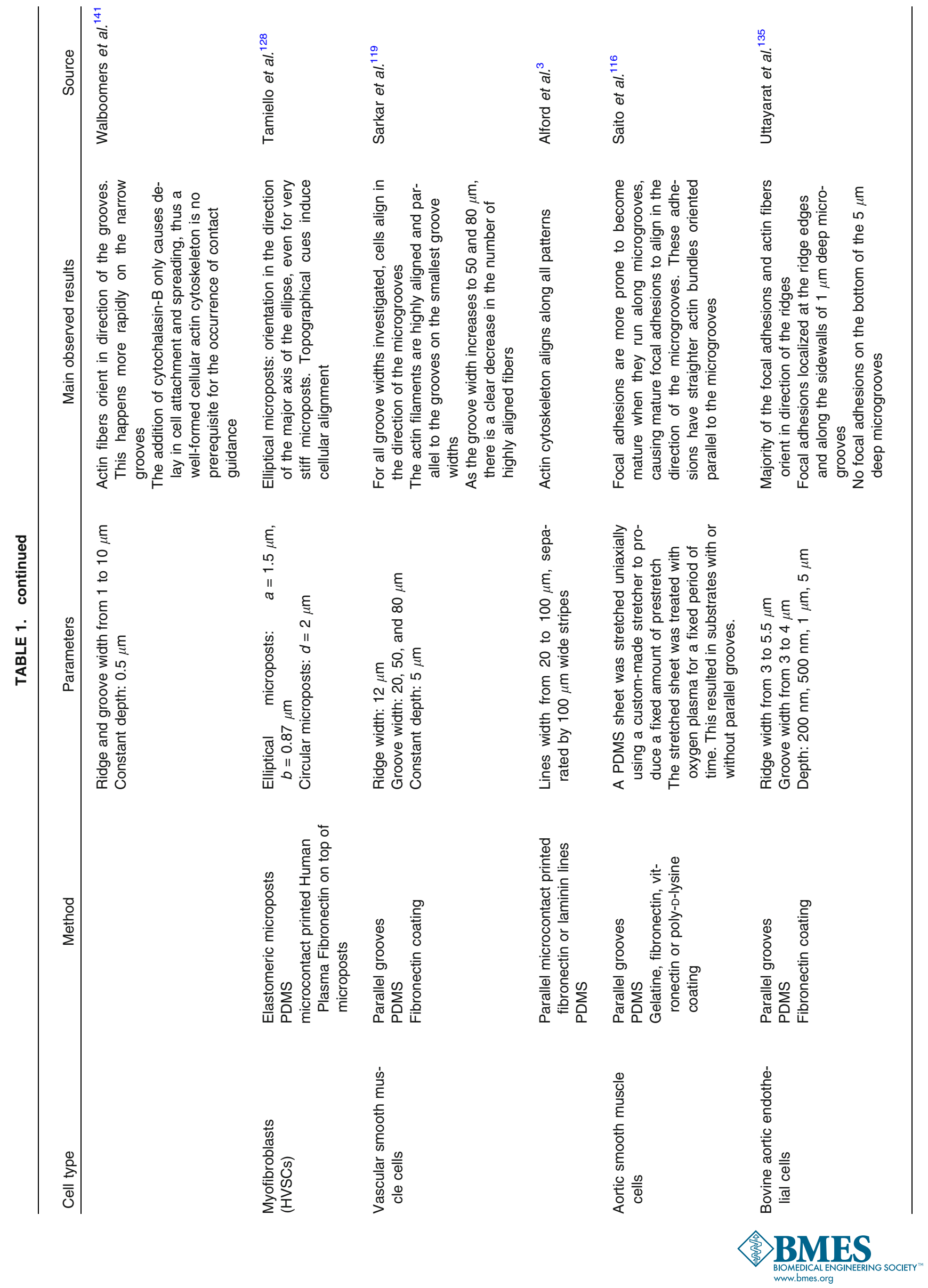




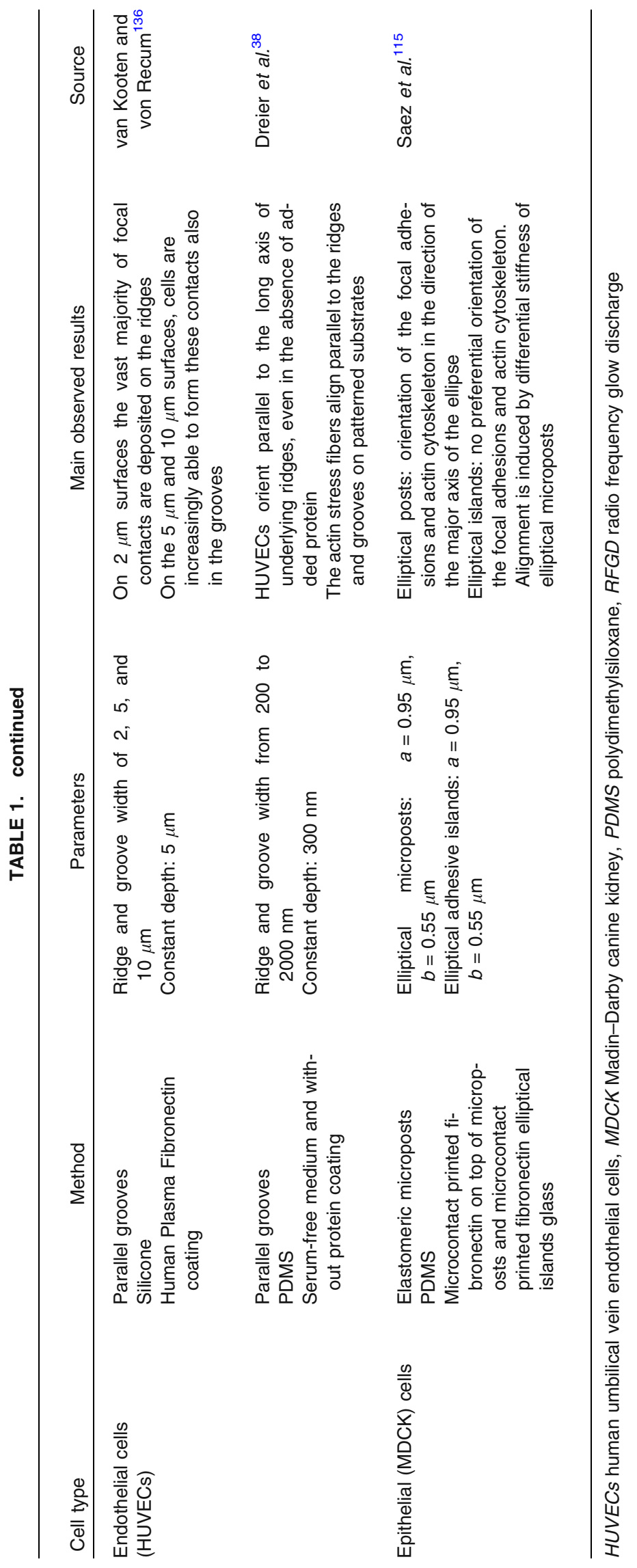


surface treatment. ${ }^{38}$ The general trend is that when either the groove width or groove height increases, the cell forms focal adhesions on the ridges and consequently orients in their direction. Next to these observations, several theoretical frameworks have been elaborated for explaining cell alignment in relation to the microgroove's parameters. The schematic representation of these theories is shown in Fig. 2.

- The mechanical restriction theory by Dunn and Heath focuses on the relative inflexibility of cytoskeletal structures as a primary regulator of cellular alignment. ${ }^{40}$ The shape of the substratum is demonstrated to impose mechanical restrictions for the formation of cytoskeletal protrusions, called filopodia, as recently shown also by Zimerman et $a l .^{167}$ and Ventre et al. ${ }^{137}$. According to this theory, the distance between the anisotropic features, either the groove width (Fig. 2b, left), on microgrooved substrates or the distance between adhesive lines on flat substrates, is the crucial factor for cell alignment. If this distance cannot be bridged by the formation of any filopodia, cells become highly polarized and elongate in the direction of the substrate anisotropy. When cells align because of this mechanism, actin filaments as well as long focal adhesions are observed in the direction of the anisotropy. These focal adhesions are usually anchored to thick stress fibres and, therefore, are presumably the focal adhesions of the actin cap stress fibers.

- The focal adhesion theory by Ohara and Buck proposes that the orientation of cells is caused by the tendency of focal adhesions to maximize their contact area. ${ }^{107}$ According to this theory, on a microgrooved substrate, focal adhesion maturation and, consequently, cell alignment occur along the ridge only if ridge width (Fig. 2b, center) is compa-

(a)
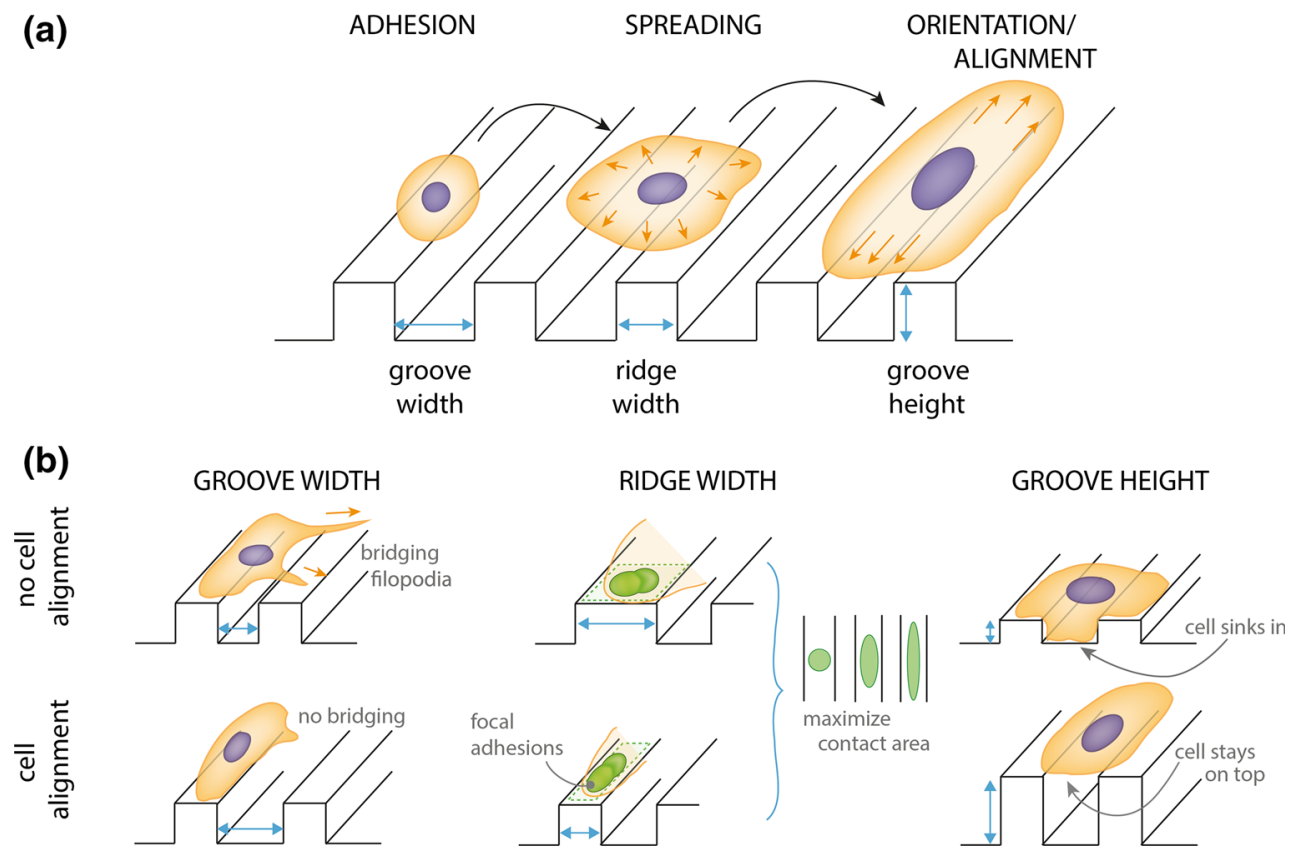

FIGURE 2. Cellular orientation response to microgrooves. (a) Schematic illustration showing the overall cellular orientation response from cell adhesion to alignment on a microgrooved substrate. At the moment the cell adheres to the microgrooved substrate, the cell undergoes spreading followed by cell alignment, i.e., orientation along the direction of the microgrooves, a phenomenon called contact guidance. The parameters characterizing the microgrooved substrate are pointed out with light blue arrows: groove width, ridge width and groove height. (b) Schematic representation of the proposed mechanisms explaining contact guidance in relation to the microgroove's parameters. (Top) No cell alignment and (bottom) cell alignment. (Left) groove width-mechanical restriction theory. When the microgrooves are too narrow, cell's filopodia succeed in bridging the space between two consecutives ridges. Therefore, the cell does not align (top). When the width of the microgrooves increases, filopodia are not able to bridge two consecutive ridges, giving the signal for cell alignment in the direction of the microgrooves (bottom). (Center) ridge width-focal adhesion theory. Ridge width influences the orientation and maturation of focal adhesions. Wide ridges do not impose geometrical confinement on the focal adhesion (green). Therefore, the maturation of the focal adhesions can occur in both directions, preventing any cell alignment (top). Narrower ridges impose geometrical confinement to the focal adhesions, which tend to maximize their contact area with the substrate. As a result, focal adhesions align and mature in the direction of the ridges, i.e., the direction of the microgrooves (bottom). (Right) groove height-discontinuity theory. For low microgrooves, the cell sinks into the microgrooves and, consequently, it does not align in direction of the microgrooves (top). For sufficiently high microgrooves, the cell senses the discontinuities of the microgrooves represented by their edges and forms focal adhesions only on the ridges. Consequently, the cell aligns in the direction of the microgrooves (bottom). Figure by Anthal Smits. 
rable to the size of a focal adhesion. An argument against this theory is the observation of focal adhesions oriented both perpendicular and parallel to ridges of the microgrooved substrate. ${ }^{105,137,167}$ However, as pointed out by Ventre et al. ${ }^{137}$ focal adhesions observed perpendicular to the ridge direction are unstable and connected to isolated actin fibers, while the focal adhesions parallel to the ridge are mature and connected to stress fibers. This ultimately guides cellular orientation in the ridge direction.

- Discontinuity theory: more recently Curtis and Clark proposed the idea that sharp discontinuities in the substrates, e.g., edges of microgrooves, induce cell alignment by triggering, first, actin condensations in these locations and, consequently, promoting focal adhesion formation at the same place. ${ }^{27}$ Despite the fact that this theory includes both the involvement of focal adhesions and actin filaments in cell alignment, it raises the question of how cells sense discontinuity, as already discussed by Curtis et al. ${ }^{28}$ Clark et al. $^{24}$ observed that by increasing the groove height (Fig. 2b, right), more cells orient in the direction of the microgrooves. Based on these observations, it is proposed that for sufficiently high microgrooves, cells are more exposed to substrate discontinuity and, as a result, align along the microgrooves.

Although these theories have shed light on the possible mechanisms behind contact guidance, the role of each individual structural component has not been fully elucidated yet. A straightforward approach to investigate the influence of the actin cytoskeleton in cellular alignment to microgrooves consists by inhibiting the actin cytoskeleton via disrupting agents, such as performed by Walboomers et al. ${ }^{141}$ and Gerecht et al. ${ }^{46}$ On one hand, Walboomers et al. observed that fibroblasts can still align along the microgrooves even if the polymerization of actin is inhibited with the use of cytochalasin-B. ${ }^{141}$ Contrarily, Gerecht et al. found that by adding actin disrupting agents to human embryo stem cells on sub-micrometer sized grooves, the morphology of the cells gets rounder. ${ }^{46}$ These results illustrate that there is no consensus yet on the role played by the actin cytoskeleton in the cellular response to contact guidance.

To unravel the relevance of each of the cellular components in the contact guidance phenomenon, a systematic approach is, in our view, needed. The various substrate features creating anisotropy need to be dissected (e.g., height, edges, biochemical patterning) and it is necessary to distinguish between substrate anisotropy by biochemical features (e.g., geometrical features given by printing of extracellular matrix pro- teins), i.e., a purely two-dimensional (2D) environment, and substrate anisotropy by topographical features (e.g., pillars, posts, microgrooves, fibers), here named two-and-a-half-dimensional (2.5D) environment. The first step towards this systematic approach, is neglecting the influence of the height of topographic features (e.g., discontinuity). Thus, as a first step, it is suggested to study contact guidance in 2D environments. Pure biochemical anisotropic features can be produced for instance by microcontact printing. According to this methodology, an elastomeric stamp of polydimethylsiloxane (PDMS) incubated with an extracellular matrix protein (e.g., fibronectin) can be used to create adhesive patterns on flat surfaces, such as glass or PDMS. The bare regions are then backfilled with a non-adhesive protein or polymer, to avoid nonspecific cell adhesion. Microcontact printing has proven to be a useful technique to adhere cells to single or multiple islands. ${ }^{20,21}$ In this way one can geometrically control cell adhesion to regulate cell functions. However, there are only limited studies where this technique has been used to induce cellular alignment via printed lines whose width is in the order of micrometers. ${ }^{3,158,167}$ In our view, this kind of studies will elucidate the precise mechanisms behind cellular alignment (Fig. 3).

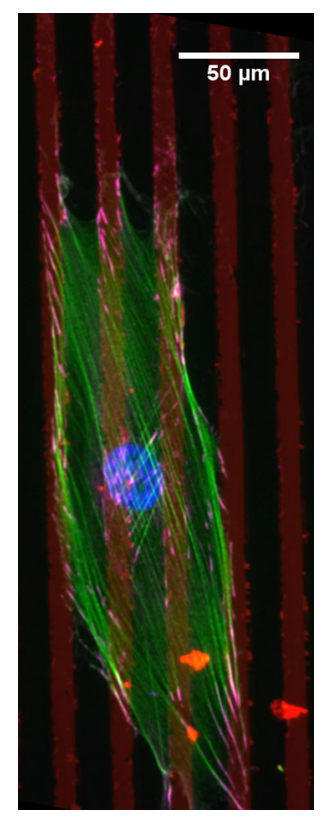

FIGURE 3. Cellular orientation response to a two-dimensional anisotropic environment. Representative microscopy image of a myofibroblast (Human Vena Saphena Cell) cultured on top of microcontact printed fibronectin (red) lines $(10 \mu \mathrm{m}$ width and $10 \mu \mathrm{m}$ spacing) on polydimethylsiloxane (PDMS). The focal adhesions are stained in magenta, the actin stress fibers in green, and the nucleus in blue. The cell orients in direction of the lines. The focal adhesions and the actin stress fibers follow cellular orientation. 


\section{Cellular Orientation Response to Uniaxial Cyclic Strain}

Cellular response to uniaxial cyclic strain is demonstrated by the dynamic reorganization and reorientation of cells and stress fibers. It has become clear that stress fibers play a crucial role in cell ability to remodel and respond appropriately to cyclic strain. Indeed, stress fiber disruption causes inhibition of cellular reorientation. ${ }^{47,59,145}$ In the $80 \mathrm{~s}$, the response of tissue cell to strain was for the first time observed and interpreted as an avoidance response to the strain of the substrate on which the cells were cultured, the so called strain avoidance response (Fig. 1b, right, Fig. 4) ${ }^{15}$ Since then, further studies have highlighted that, on 2D substrates, cell reorientation occurs at angles (nearly) perpendicular to the stretch direction, i.e., the direction of minimal substrate deformation (Fig. 4). In the last decades, several studies have been carried out in order to quantify and unravel the mechanisms of this phenomenon. Stretch avoidance appears to be a behavior belonging to many kinds of tissue cells, ranging from endothelial cells, ${ }^{6,31,66,73,74,82,101,144,145,157,161}$ to fibroblasts ${ }^{9,70,100}$ and smooth muscle cells. ${ }^{32,71,126}$ However, the dependence of such response on the spatiotemporal parameters of the cyclic stimulation, such as frequency, ${ }^{66,70,88}$ magnitude, ${ }^{9,32,66,71,73,144,157}$ strain rate, ${ }^{67,82,100,133}$ duration, ${ }^{160,161,165}$ or even the combination of some of those, ${ }^{148}$ makes any attempt to correlate the effects of these factors with cellular response unsuccessful. Moreover stretch avoidance response seems to be cell type dependent and a minimal strain amplitude, ${ }^{6,32,100}$ frequency $^{66,67,70}$ or cell contractile status ${ }^{41}$ may be required for the response to occur. Summary of studies about cells of the cardiovascular system (fibroblasts, tissue cells, endothelial and progenitor cells) and stress fiber response to uniaxial cyclic stretch are reported in Table 2.

Most of these studies are carried out with custombuilt devices for which an accurate and rigorous strain characterization is needed, but often overlooked. These devices are made of motorized stages capable to stretch silicone membranes coated with extracellular matrix proteins such as fibronectin or collagen. Given the mechanical properties of the elastomeric materials, once the membrane is stretched along one direction, it contracts in the perpendicular direction (Poisson's effect). New commercially available devices have been designed to avoid this drawback (FlexCell ${ }^{5,88,126}$ and STREX $^{133}$ ). Nevertheless, the use of such diverse instrumentations cannot help to distinguish between the impacts of the different factors. Moreover, the interference of signaling mechanisms cannot be excluded when different coatings are employed. Altogether, controlled experimental conditions are needed towards a comprehensive understanding of cell reorientation.

Efforts to unravel the spatiotemporal dynamics of cellular adaptations especially at the level of stress fibers are still limited. Most of the observations come from a state-to-state like manner, making use of fixed cells that do not allow observations of subcellular dynamics. The technological challenges that must be overcome include the use of actin stress fiber probes that do not interfere with the dynamics of actin polymerization and the mechanical properties. ${ }^{36}$ Moreover, the timescale of actin reorganization pushes further the experimental limits.
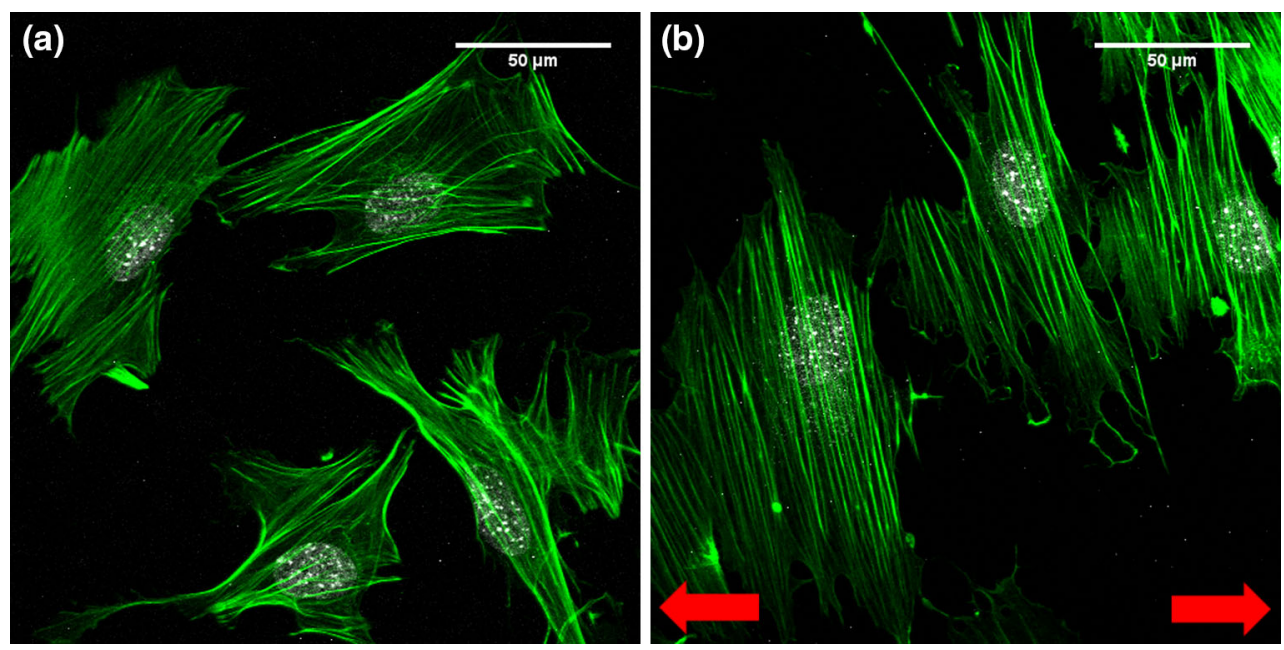

FIGURE 4. Cellular orientation response to uniaxial cyclic strain. (a) Mouse embryonic fibroblasts (MEFs) cultured in static conditions for $6 \mathrm{~h}$ on a homogenously fibronectin-coated silicone membrane and stained for actin stress fibers (green) and nucleus (white). Cells and actin stress fibers are oriented randomly. (b) MEFs after $24 \mathrm{~h}$ of uniaxial cyclic strain $(7 \%, 0.5 \mathrm{~Hz})$. Cells and stress fiber are oriented almost perpendicularly to the strain direction (red arrows). This response is called strain avoidance. 
TAMiello et $a l$.

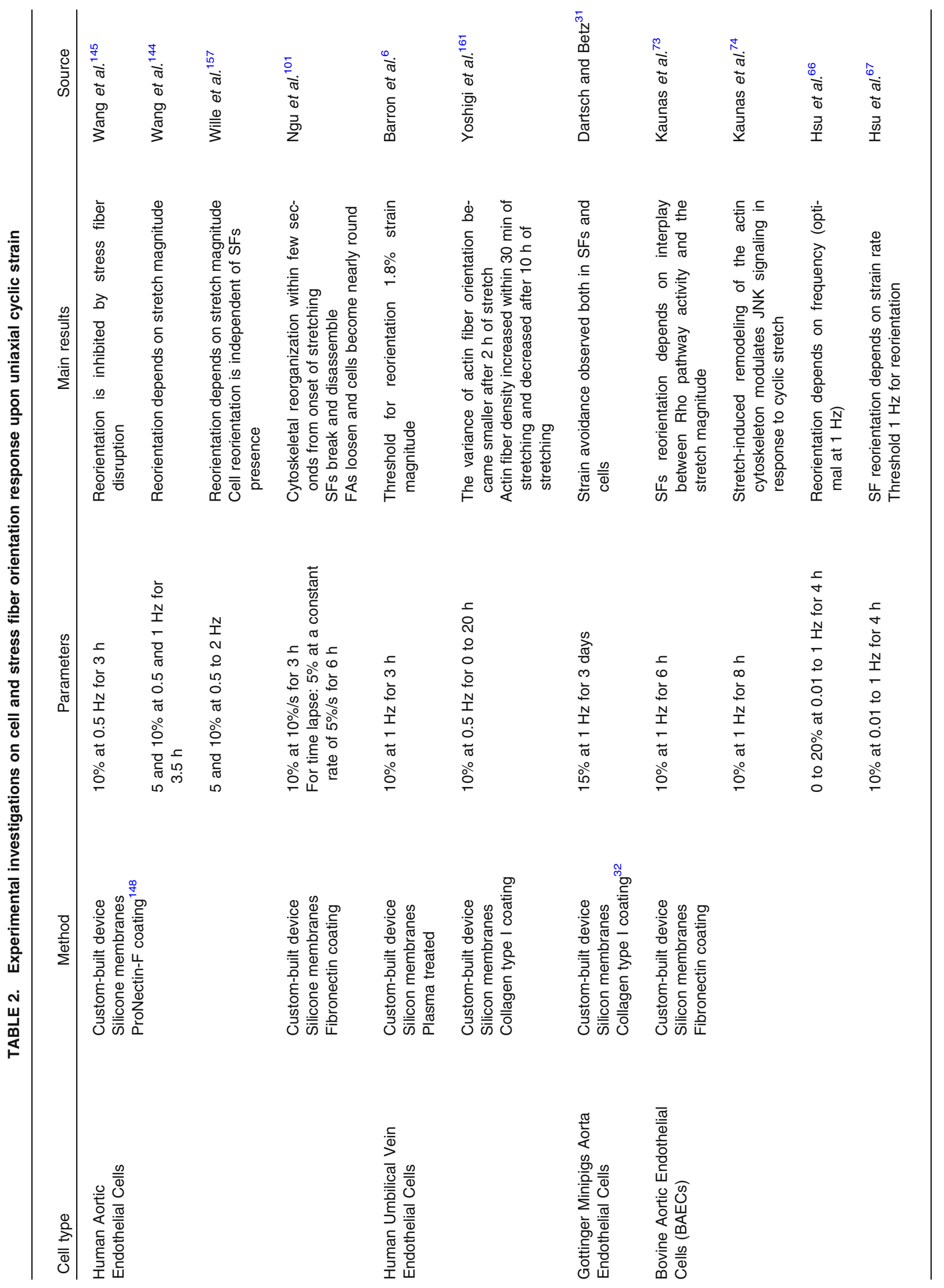


Understanding Cellular Orientation Responses

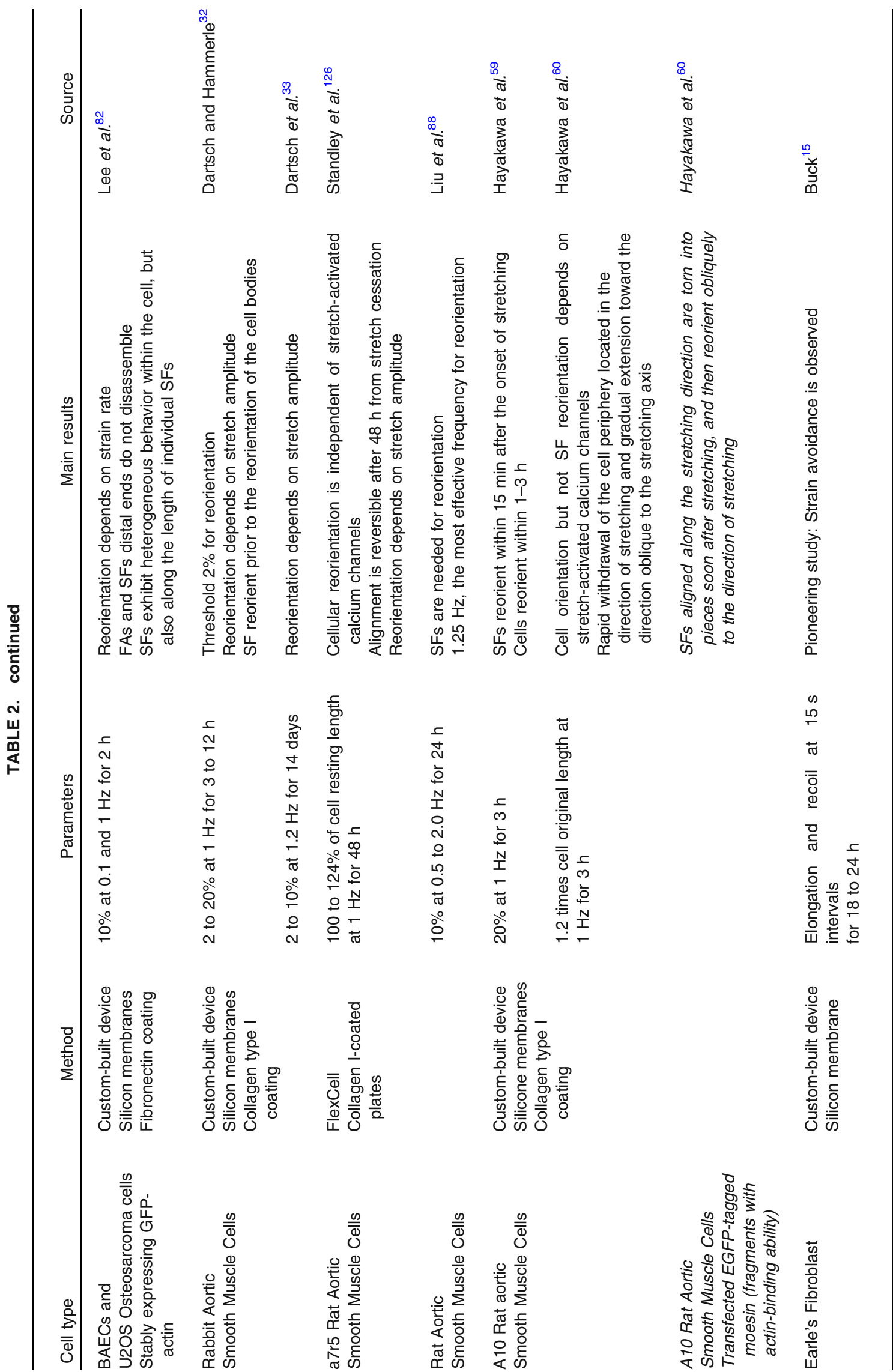


TAMiello $e t$ al.

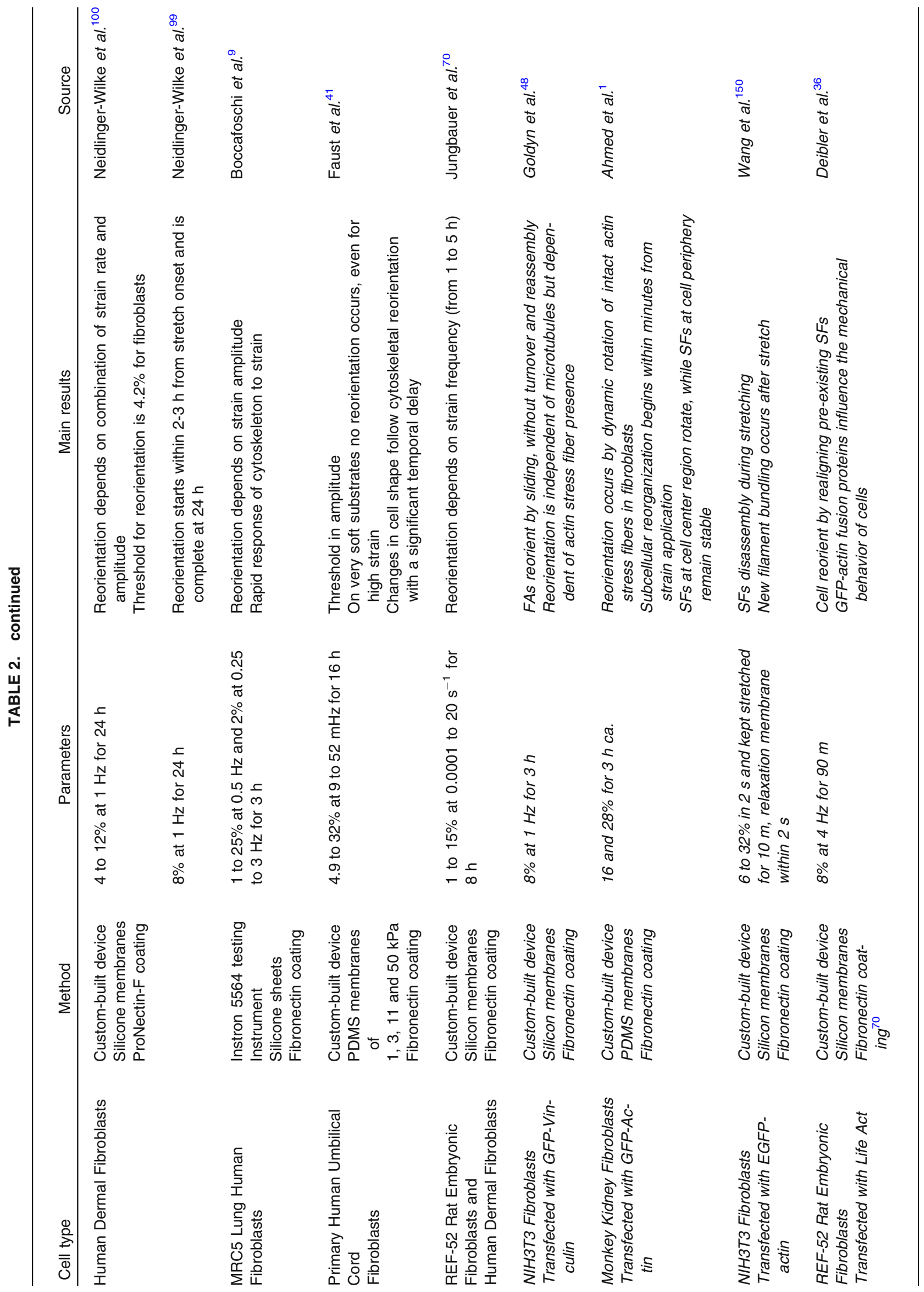




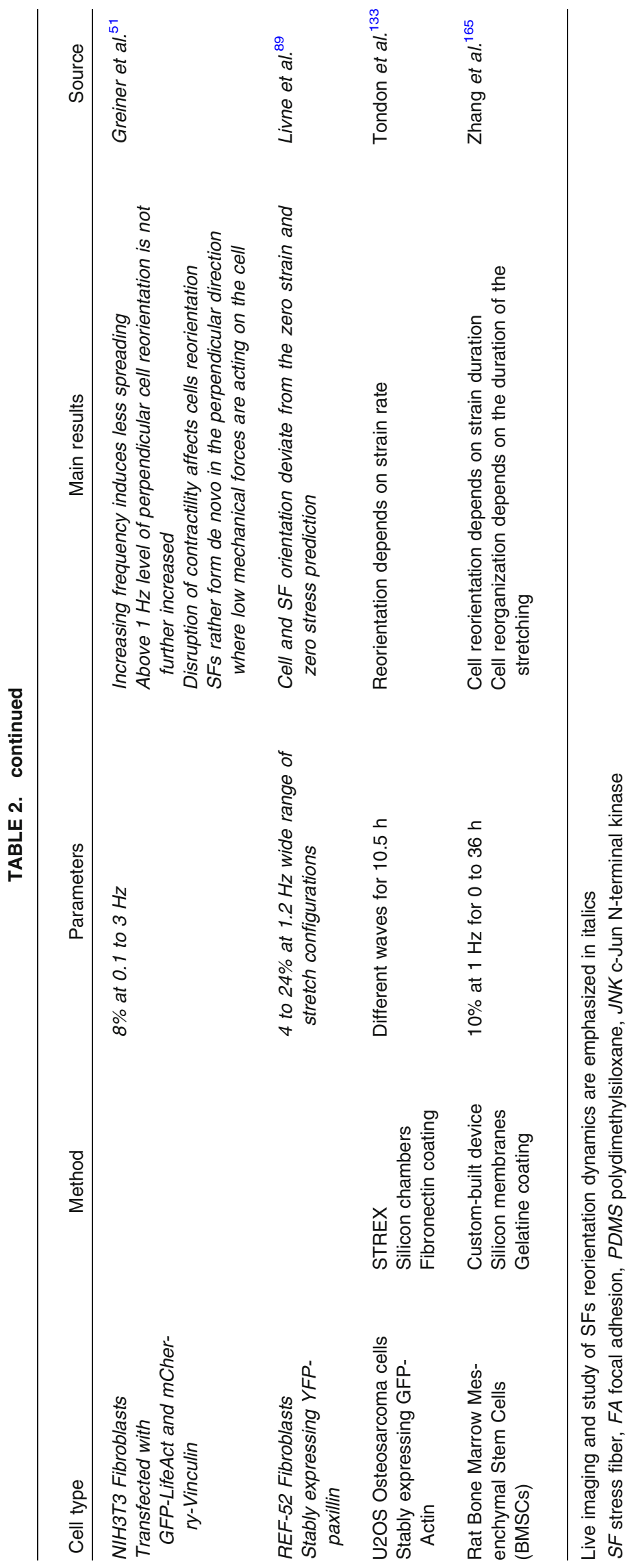


From time-lapse studies, it has been established that cells become nearly round in the first phases of reorientation and, subsequently elongate along the strain avoidance direction. ${ }^{59,70,101}$ During this second phase, a process of reinforcement and repair of the stress fiber strain sites occurs. Zyxin is recruited at strain-induced damage sites of stress fibers and subsequently activates actin cytoskeleton repair and reinforcement. ${ }^{55,84,123,124,162}$ In terms of temporal dynamics, stress fibers significantly anticipate cell overall reorientation. Stress fiber reorganization response occurs within the first minutes from the onset of the cyclic strain stimulation, while complete cell reorientation is seen in the time range of hours. ${ }^{32,59}$ In 2001, Hayakawa et al. observed in rat smooth muscle cells the breakdown of stress fibers aligned along the stretching direction soon after the start of the mechanical stimulation, followed by stress fiber reorientation at an oblique angle with respect to the axis of stretching. ${ }^{60}$ Similar observations were reported by $\mathrm{Ngu}$ et al. in bovine endothelial aortic cells. ${ }^{101}$ Also, the investigation of Lee et al. pointed out that bovine aortic endothelial cell reorientation involved the disassembly of the stress fiber proximal section (far from the focal adhesions) and de-novo formation of stress fiber at a reoriented angle with comparatively little focal adhesion turnover. ${ }^{82}$ These studies suggest that reorientation of stress fiber takes place through stress fiber turnover and re-assembly. However, there is also another line of evidence which suggests that stress fiber turnover might occur via focal adhesion sliding and consequent stress fiber rotation. Deibler et al. ${ }^{36}$ demonstrated that rat embryonic fibroblasts reorient by realigning pre-existing stress fiber while Goldyn et l. $^{48}$ tracked the dramatical sliding of focal adhesions induced by uniaxial cyclic strain in NIH3T3 fibroblasts. Most probably, the aforementioned mechanisms are not mutually exclusive. Still, the challenge for the future remains to uncover the precise mechanisms of stress fiber and cell reorientation, by focusing on the heterogeneity observed not only on subcellular locations but also along the same stress fibers. ${ }^{1,82}$

A number of theoretical models have been elaborated in the endeavor to describe the relationship between the actin cytoskeleton reorganization and the uniaxial cyclic strain acting on cells. In 2000, Wang et al. proposed that stress fibers tend to orient in the direction of minimal normal strain, where the unperturbed state is maintained. ${ }^{143}$ Other models, mostly based on the molecular aspects of stress fiber assembly, ${ }^{72,106,152}$ were developed based on the same approach. Instead, the work of De et al. predicts stress fiber orientation in the minimal matrix stress direction using a coarse-grained model of cells approximated as single force dipoles. ${ }^{34}$ While consistency between the predictions of these models and experimental results was proven in many studies, recently, Livne et al. have found significant deviation between their results and the theoretical predictions proposed by the existing models. ${ }^{89}$ By investigating strain avoidance response over a wide range of stretch configurations, they demonstrated that stress fiber reorganization does not coincide with the direction of minimal strain or stress of the substrate. Therefore, they developed a new theoretical approach based on the molecular and physical properties of the stress fiber-focal adhesion system. Yet, it remains to be tested whether this model is cell-type independent.

\section{Cellular Orientation Response to Combined Substrate Anisotropy and Uniaxial Cyclic Strain}

From the previous paragraphs it appears that cell and stress fiber orientation can be influenced by anisotropic cues or by imposing uniaxial cyclic strain on cell growth substrates. This legitimates to ask what the influence of anisotropic cues and cyclic strain is when these cues are applied in combination and along the same direction. This simultaneous stimulation, theoretically, would lead to competing stimuli for cell reorientation. An overview of the studies conducted applying anisotropic cues and uniaxial cyclic strain are reported in Table 3. We have considered all cell types, as the number of these studies is limited.

By using microgrooves integrated in a custom-built stretching device, Wang and Grood demonstrated that micro-topography generally overrules strain avoidance, i.e., adherent cells maintain the original orientation imposed by the microgrooves, even if strain stimulation occurs along the same direction. ${ }^{146,147,151}$ Prodanov et al. have added to this evidence that cellular response might be influenced by the dimension of the anisotropic textures. ${ }^{12}$ They showed that osteoblasts plated on nanogrooves and subjected to cyclic strain responded by strain avoidance, while on micro-sized features, they remained aligned with the anisotropy of the substrate. Next to this, it has been observed by Ahmed et al. that topographical cues combined with cyclic strain can have distinct impact on stress fibers as compared to cell body reorientation response. ${ }^{2}$ In their study, myoblasts were confined on substrates patterned with parallel fibronectin lines (widths comparable to cell size) and exposed to uniaxial cyclic stretch. It appeared that, while cell bodies remained confined on the micropatterned lines, stress fiber succeeded in reorganizing perpendicular to the strain direction. This points to different mechanisms involved in strain and anisotropy sensing. 


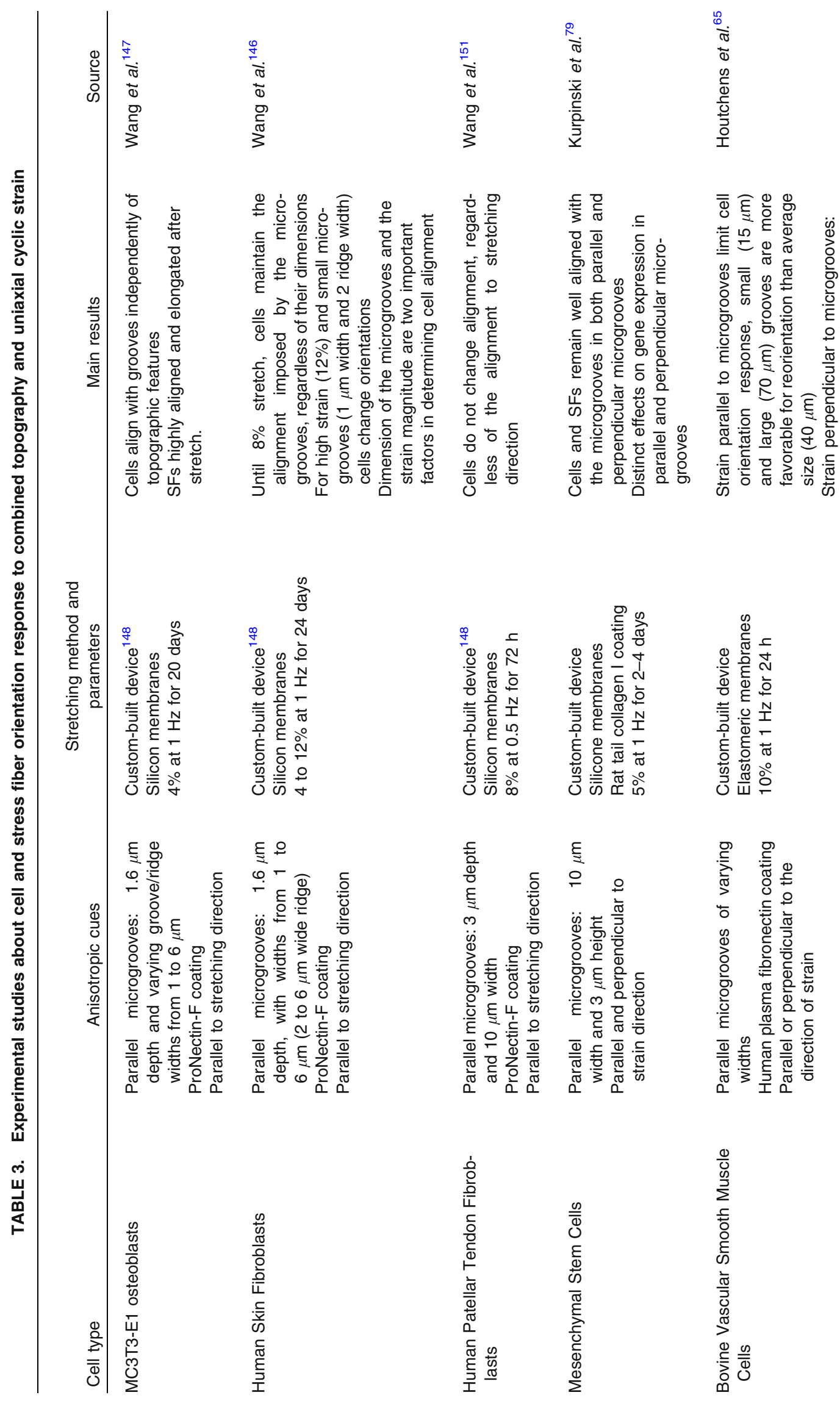

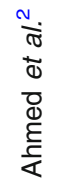

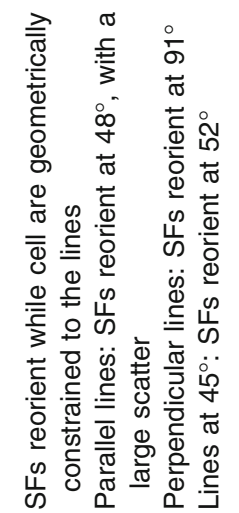




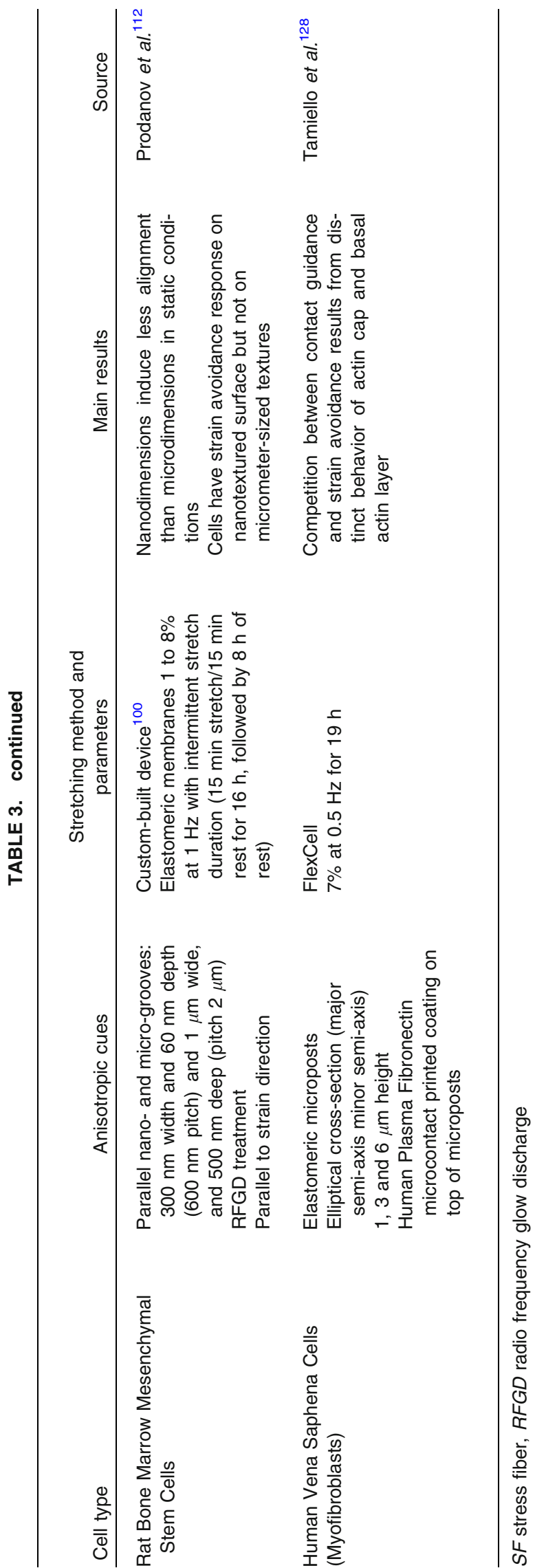

Recently, a study from our group has provided further insight on the mechanisms underlying stress fiber response to combined cyclic strain and anisotropic cues. It was observed that distinct responses occur at the actin cap and basal layer (Fig. 5). The actin cap stress fibers clearly tend to neglect the topographical cues and respond to strain, while the basal actin fibers remain aligned with the topography of the substrate. ${ }^{128}$ These findings provided evidence that cellular response to anisotropy of the substrate and cyclic strain is the complex integration of subcellular structural responses. Nevertheless, most of the mentioned studies reported on cell and stress fiber orientation but neglected the response of crucial structures such as focal adhesions.

Altogether, the examples reported above show that, although general indication exists that anisotropic cues modulate cell and stress fiber orientation response to uniaxial cyclic strain, a deeper understanding of the phenomenon is still needed. Detailed quantification of stress fiber reorientation dynamics at the subcellular level upon presentation of simultaneous anisotropic and cyclic strain cues would be of great benefit for unraveling the temporal dynamics of the processes involved in cellular adaptation.

\section{SUMMARY AND OUTLOOK}

A deep understanding of the mechanisms by which biophysical cues regulate cellular orientation is fundamental for cardiovascular regeneration strategies, such as biomaterial-based in situ engineering approaches that need to guide and control cell and tissue organization for proper tissue functioning. In particular, cell alignment is a primary aim of regeneration of cardiovascular tissues, as controlled cellular organization is essential for matching native tissue micro architecture and functionality. ${ }^{110}$

In this work, we provide an overview of the knowledge obtained in the last decades about the components of the structural mechanotransduction pathway, an interconnected chain of proteins implicated in force propagation from the extracellular environment to downstream targets such as the nucleus and gene expression regulation. We also report on the current understanding of cellular orientation responses induced by the application of anisotropic cues and uniaxial cyclic strain focusing on the experimental evidence obtained with in vitro studies using single cell observations. The development of in vivo-like micro-devices has enabled researchers to perform experimental studies under controlled conditions, in the effort to uncover the link between applied biophysical cues and cellular response. Still, 

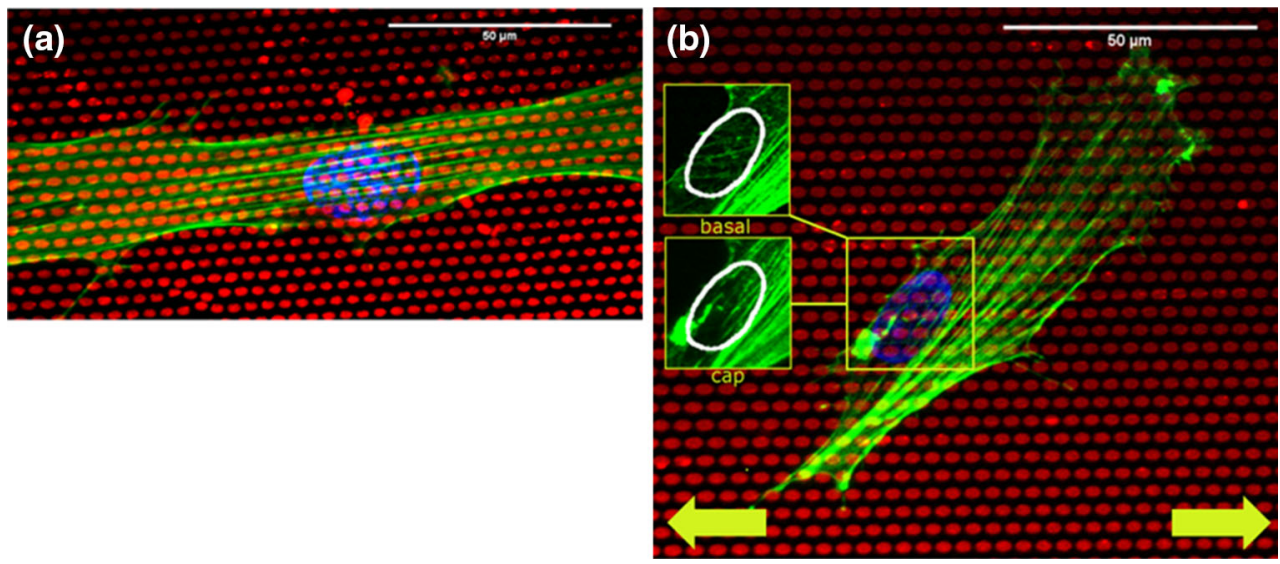

FIGURE 5. Cellular orientation response to combined substrate anisotropy and uniaxial cyclic strain. (a) Myofibroblasts (Human Vena Saphena Cells) cultured on top of fibronectin-coated elliptical microposts (red) in static conditions for $6 \mathrm{~h}$. The stress fibers, colored in green, orient along the substrate anisotropy, i.e., along the micropost major axis. The nucleus is shown in blue. (b) The system made of elliptical microposts can be stretched along the micropost major axis (horizontal direction, yellow arrows). The use of this model system revealed that, the orientation response of myofibroblasts exposed to substrate anisotropy and strain $(19 \mathrm{~h}, 7 \%, 0.5 \mathrm{~Hz})$ along the same direction is determined by the distinct response of the actin stress fibers running on top of the nucleus (inset cap) and the ones present underneath the nucleus and connected to the microposts (inset basal). While the cap actin fibers respond by strain avoidance, the basal stress fibers tend to follow the direction on the micropost major axis. ${ }^{118}$

the large body of knowledge generated by using such diverse approaches and the cell-type dependence of the results complicate the attempt of unifying the knowledge.

All in all, the above examples demonstrate that an intact structural mechanotransduction pathway plays a crucial role in the control of normal cellular functionality. Nevertheless, in order to move forward towards understanding the complex interplay between cellular mechanoresponses and biophysical properties of the micro-environment, it is important to identify the main scientific challenges.

Firstly, further research is required to achieve an indepth understanding of the role of the structural mechanotransduction pathway. It is necessary to determine the completeness of our current understanding of such a pathway. More importantly, it is crucial to identify the relevance of individual and combined components of this pathway for controlling cell orientation. An interesting strategy within consists in considering cells with defected structural connections or knock-out cellular models as tools for novel investigations. A first attempt has been conducted by Tamiello et al. (unpublished) by using actin cap-lacking fibroblasts. In this study the relevance of the actin cap in the response to anisotropic cues and strain stimulation was studied by exposing the cells to both cues, applied separately and in combination. Interestingly, since these knock-out cells have been obtained by elimination A-type lamins, they represent also a useful model for studying the development of the family of mechanotransduction diseases named laminopathies. In general, mechanotransduction studies on cells from diseased patients will not only advance our understanding of the relevance of the structural connection in the cellular mechanoresponse, but also elucidate whether diseases/disorders of mechanotransduction primarily result from structural defects, impaired biochemical signaling or a synergy between the two mechanotransduction processes. Progress in this field will eventually lead to the design of effective regenerative strategies for a variety of diseases arising for mechanotransduction defects. ${ }^{69}$

Secondly, future studies should focus on designing a unified systematic approach for studying cellular responses to individual biophysical cues. This is needed in order to get quantifiable measurements of the effects of the various parameters of the micro-environment on cell and stress fiber orientation, for instance. Such a simplified approach will enable the integration of the overwhelming amount of information obtained using an array of diverse devices and, consequently, enhance our knowledge about the influence of biophysical cues on cellular alignment. Once cellular responses to individual cues are established, the next step we envision is to develop integrative approaches to study cellular response to combined cues, in a more tissue-like context. This is necessary in order to unravel whether a signaling hierarchy coming from distinct cues exists. Another suggestion is to develop high-throughput systems based on the simplified systematic approach in order to screen the effects of individual and combined biophysical cues on relevant cell outputs (orientation, force, proliferation etc.). Such a systematic approach can give reliable inputs for computational models in cell mechanics to interpret experimental observations 
and elucidate main governing processes of cellular mechanoresponse.

Finally, more investigations are necessary to obtain an in-depth understanding of the mechanisms underlying cell mechanoresponse. To address issues on how cells integrate and transduce physical signals, it is crucial to develop live imaging techniques to analyze the structural responses at subcellular level with higher spatial and temporal resolutions. The next big technological challenge will in the application of these tools to more complex environments, such as three-dimensional (3D) substrates. These substrates are of special interest because they mimic more closely the physiological environment of tissue cells. Recent evidences suggest that cellular behaviors in $3 \mathrm{D}$ environments differ from observations obtained employing two-dimensional (2D) environments.

As the intricate aspects about the cellular mechanoresponse become to be better characterized, it may become possible to open new avenues for controlling the way in which cell interact and respond with their physical micro-environment. We envision this is the way forward to effectively elaborate targeted strategies for tissue regeneration and therapeutical approaches.

\section{CONFLICT OF INTEREST}

Chiara Tamiello, Antonetta Buskermolen, Frank Baaijens, Jos Broers and Carlijn Bouten declare that they have no conflict of interest.

\section{HUMAN AND ANIMAL RIGHTS}

No human and animal studies were carried out by the authors of this article.

\section{OPEN ACCESS}

This article is distributed under the terms of the Creative Commons Attribution 4.0 International License (http://creativecommons.org/licenses/by/4.0/), which permits unrestricted use, distribution, and reproduction in any medium, provided you give appropriate credit to the original author(s) and the source, provide a link to the Creative Commons license, and indicate if changes were made.

\section{REFERENCES}

${ }^{1}$ Ahmed, W. W., M. H. Kural, and T. A. Saif. A novel platform for in situ investigation of cells and tissues under mechanical strain. Acta Biomater. 6:2979-2990, 2010.
${ }^{2}$ Ahmed, W. W., T. Wolfram, A. M. Goldyn, K. Bruellhoff, B. A. Rioja, M. Moller, J. P. Spatz, T. A. Saif, J. Groll, and R. Kemkemer. Myoblast morphology and organization on biochemically micro-patterned hydrogel coatings under cyclic mechanical strain. Biomaterials 31:250-258, 2010.

${ }^{3}$ Alford, P. W., A. P. Nesmith, J. N. Seywerd, A. Grosberg, and K. K. Parker. Vascular smooth muscle contractility depends on cell shape. Integr. Biol. (Camb.) 3:1063-1070, 2011.

${ }^{4}$ Alon, R., and M. L. Dustin. Force as a facilitator of integrin conformational changes during leukocyte arrest on blood vessels and antigen-presenting cells. Immunity. 26:17-27, 2007.

${ }^{5}$ Banes, A. J., J. Gilbert, D. Taylor, and O. Monbureau. A new vacuum-operated stress-providing instrument that applies static or variable duration cyclic tension or compression to cells in vitro. J. Cell Sci. 75:35-42, 1985.

${ }^{6}$ Barron, V., C. Brougham, K. Coghlan, E. McLucas, D. O'Mahoney, C. Stenson-Cox, and P. E. McHugh. The effect of physiological cyclic stretch on the cell morphology, cell orientation and protein expression of endothelial cells. J. Mater. Sci. Mater. Med. 18:1973-1981, 2007.

${ }^{7}$ Bettinger, C. J., R. Langer, and J. T. Borenstein. Engineering substrate topography at the micro- and nanoscale to control cell function. Angew. Chem. Int. Ed Engl. 48:5406-5415, 2009.

${ }^{8}$ Bione, S., E. Maestrini, S. Rivella, M. Mancini, S. Regis, G. Romeo, and D. Toniolo. Identification of a novel xlinked gene responsible for emery-dreifuss muscular-dystrophy. Nat. Genet. 8:323-327, 1994.

${ }^{9}$ Boccafoschi, F., M. Bosetti, S. Gatti, and M. Cannas. Dynamic fibroblast cultures: response to mechanical stretching. Cell Adh. Migr. 1:124-128, 2007.

${ }^{10}$ Borejdo, J., and S. Burlacu. Measuring orientation of actinfilaments within a cell-orientation of actin in intestinal microvilli. Biophysical Journal 65:300-309, 1993.

${ }^{11}$ Britland, S., H. Morgan, B. Wojiak-Stodart, M. Riehle, A. Curtis, and C. Wilkinson. Synergistic and hierarchical adhesive and topographic guidance of BHK cells. Exp. Cell Res. 228:313-325, 1996.

${ }^{12}$ Broers, J. L., E. A. Peeters, H. J. Kuijpers, J. Endert, C. V. Bouten, C. W. Oomens, F. P. Baaijens, and F. C. Ramaekers. Decreased mechanical stiffness in LMNA-/cells is caused by defective nucleo-cytoskeletal integrity: implications for the development of laminopathies. Hum. Mol. Genet. 13:2567-2580, 2004.

${ }^{13}$ Broers, J. L., F. C. Ramaekers, G. Bonne, R. B. Yaou, and C. J. Hutchison. Nuclear lamins: laminopathies and their role in premature ageing. Physiol. Rev. 86:967-1008, 2006.

${ }^{14}$ Brosig, M., J. Ferralli, L. Gelman, M. Chiquet, and R. Chiquet-Ehrismann. Interfering with the connection between the nucleus and the cytoskeleton affects nuclear rotation, mechanotransduction and myogenesis. Int. J. Biochem. Cell Biol. 42:1717-1728, 2010.

${ }^{15}$ Buck, R. C. Reorientation response of cells to repeated stretch and recoil of the substratum. Exp. Cell Res. 127:470-474, 1980.

${ }^{16}$ Burke, B., and C. L. Stewart. The laminopathies: the functional architecture of the nucleus and its contribution to disease. Annu. Rev. Genom. Hum. Genet. 7:369-405, 2006.

${ }^{17}$ Burridge, K., and E. S. Wittchen. The tension mounts: stress fibers as force-generating mechanotransducers. $J$. Cell Biol. 200:9-19, 2013.

${ }^{18}$ Chambliss, A. B., S. B. Khatau, N. Erdenberger, D. K. Robinson, D. Hodzic, G. D. Longmore, and D. Wirtz. The 
LINC-anchored actin cap connects the extracellular milieu to the nucleus for ultrafast mechanotransduction. Sci. Rep. 3:1087, 2013.

${ }^{19}$ Chancellor, T. J., J. Lee, C. K. Thodeti, and T. Lele. Actomyosin tension exerted on the nucleus through nesprin-1 connections influences endothelial cell adhesion, migration, and cyclic strain-induced reorientation. Biophys. J. 99:115-123, 2010.

${ }^{20}$ Chen, C. S., J. L. Alonso, E. Ostuni, G. M. Whitesides, and D. E. Ingber. Cell shape provides global control of focal adhesion assembly. Biochem. Biophys. Res. Commun. 307:355-361, 2003.

${ }^{21}$ Chen, C. S., M. Mrksich, S. Huang, G. M. Whitesides, and D. E. Ingber. Geometric control of cell life and death. Science 276:1425-1428, 1997.

${ }^{22}$ Choi, C. K., M. Vicente-Manzanares, J. Zareno, L. A. Whitmore, A. Mogilner, and A. R. Horwitz. Actin and alpha-actinin orchestrate the assembly and maturation of nascent adhesions in a myosin II motor-independent manner. Nat. Cell Biol. 10:1039-1050, 2008.

${ }^{23}$ Chung, A. S., and N. Ferrara. Developmental and pathological angiogenesis. Annu. Rev. Cell Dev. Biol. 27:563-584, 2011.

${ }^{24}$ Clark, P., P. Connolly, A. S. Curtis, J. A. Dow, and C. D. Wilkinson. Topographical control of cell behaviour: II. Multiple grooved substrata. Development 108:635-644, 1990.

${ }^{25}$ Cook, J. R., L. Carta, L. Benard, E. R. Chemaly, E. Chiu, S. K. Rao, T. G. Hampton, P. Yurchenco, K. D. Costa, R. J. Hajjar, and F. Ramirez. Abnormal muscle mechanosignaling triggers cardiomyopathy in mice with Marfan syndrome. J. Clin. Invest 124:1329-1339, 2014.

${ }^{26}$ Crisp, M., Q. Liu, K. Roux, J. B. Rattner, C. Shanahan, B. Burke, P. D. Stahl, and D. Hodzic. Coupling of the nucleus and cytoplasm: role of the LINC complex. J. Cell Biol. 172:41-53, 2006.

${ }^{27}$ Curtis, A. S., and P. Clark. The effects of topographic and mechanical properties of materials on cell behavior. $C R C$ Rev. Biocompatibility 5:343-362, 1990.

${ }^{28}$ Curtis, A., and C. Wilkinson. Topographical control of cells. Biomaterials 18:1573-1583, 1997.

${ }^{29}$ Dahl, K. N., A. J. Ribeiro, and J. Lammerding. Nuclear shape, mechanics, and mechanotransduction. Circ. Res. 102:1307-1318, 2008.

${ }^{30}$ Dalby, M. J. Topographically induced direct cell mechanotransduction. Med. Eng Phys. 27:730-742, 2005.

${ }^{31}$ Dartsch, P. C., and E. Betz. Response of cultured endothelial cells to mechanical stimulation. Basic Res. Cardiol. 84:268-281, 1989.

${ }^{32}$ Dartsch, P. C., and H. Hammerle. Orientation response of arterial smooth muscle cells to mechanical stimulation. Eur. J. Cell Biol. 41:339-346, 1986.

${ }^{33}$ Dartsch, P. C., H. Hammerle, and E. Betz. Orientation of cultured arterial smooth muscle cells growing on cyclically stretched substrates. Acta Anat. (Basel) 125:108-113, 1986.

${ }^{34}$ De, R., and S. A. Safran. Dynamical theory of active cellular response to external stress. Phys. Rev. E. Stat. Nonlinear Soft. Matter Phys. 78:031923, 2008.

${ }^{35}$ Dechat, T., K. Pfleghaar, K. Sengupta, T. Shimi, D. K. Shumaker, L. Solimando, and R. D. Goldman. Nuclear lamins: major factors in the structural organization and function of the nucleus and chromatin. Genes Dev. 22:832853, 2008.

${ }^{36}$ Deibler, M., J. P. Spatz, and R. Kemkemer. Actin fusion proteins alter the dynamics of mechanically induced cytoskeleton rearrangement. PLoS One 6:e22941, 2011.
${ }^{37}$ den Braber, E. T., J. E. de Ruijter, L. A. Ginsel, A. F. von Recum, and J. A. Jansen. Quantitative analysis of fibroblast morphology on microgrooved surfaces with various groove and ridge dimensions. Biomaterials 17:2037-2044, 1996.

${ }^{38}$ Dreier, B., J. Z. Gasiorowski, J. T. Morgan, P. F. Nealey, P. Russell, and C. J. Murphy. Early responses of vascular endothelial cells to topographic cues. Am. J. Physiol Cell Physiol 305:C290-C298, 2013.

${ }^{39}$ Dunn, G. A., and A. F. Brown. Alignment of fibroblasts on grooved surfaces described by a simple geometric transformation. J. Cell Sci. 83:313-340, 1986.

${ }^{40}$ Dunn, G. A., and J. P. Heath. A new hypothesis of contact guidance in tissue cells. Exp. Cell Res. 101:1-14, 1976.

${ }^{41}$ Faust, U., N. Hampe, W. Rubner, N. Kirchgessner, S. Safran, B. Hoffmann, and R. Merkel. Cyclic stress at $\mathrm{mHz}$ frequencies aligns fibroblasts in direction of zero strain. PLoS One 6:e28963, 2011.

${ }^{42}$ Feinberg, A. W., P. W. Alford, H. Jin, C. M. Ripplinger, A. A. Werdich, S. P. Sheehy, A. Grosberg, and K. K. Parker. Controlling the contractile strength of engineered cardiac muscle by hierarchal tissue architecture. Biomaterials 33:5732-5741, 2012.

${ }^{43}$ Freedman, B. R., N. D. Bade, C. N. Riggin, S. Zhang, P. G. Haines, K. L. Ong, and P. A. Janmey. The (dys)functional extracellular matrix. Biochim. Biophys. Acta 1853:3153-3164, 2015.

${ }^{44}$ Geiger, B., J. P. Spatz, and A. D. Bershadsky. Environmental sensing through focal adhesions. Nat. Rev. Mol. Cell Biol. 10:21-33, 2009.

${ }^{45}$ Geiger, B., and K. M. Yamada. Molecular architecture and function of matrix adhesions. Cold Spring Harb. Perspect. Biol. 3:a005033, 2011.

${ }^{46}$ Gerecht, S., C. J. Bettinger, Z. Zhang, J. T. Borenstein, G. Vunjak-Novakovic, and R. Langer. The effect of actin disrupting agents on contact guidance of human embryonic stem cells. Biomaterials 28:4068-4077, 2007.

${ }^{47}$ Goldyn, A. M., P. Kaiser, J. P. Spatz, C. Ballestrem, and R. Kemkemer. The kinetics of force-induced cell reorganization depend on microtubules and actin. Cytoskeleton (Hoboken.) 67:241-250, 2010.

${ }^{48}$ Goldyn, A. M., B. A. Rioja, J. P. Spatz, C. Ballestrem, and R. Kemkemer. Force-induced cell polarisation is linked to RhoA-driven microtubule-independent focal-adhesion sliding. J. Cell Sci. 122:3644-3651, 2009.

${ }^{49}$ Gonzalez-Suarez, I., A. B. Redwood, and S. Gonzalo. Loss of A-type lamins and genomic instability. Cell Cycle 8:3860-3865, 2009.

${ }^{50}$ Gregor, M., S. Osmanagic-Myers, G. Burgstaller, M. Wolfram, I. Fischer, G. Walko, G. P. Resch, A. Jorgl, H. Herrmann, and G. Wiche. Mechanosensing through focal adhesion-anchored intermediate filaments. FASEB J. 28:715-729, 2014.

${ }^{51}$ Greiner, A. M., H. Chen, J. P. Spatz, and R. Kemkemer. Cyclic tensile strain controls cell shape and directs actin stress fiber formation and focal adhesion alignment in spreading cells. PLoS One 8:e77328, 2013.

${ }^{52}$ Grenier, G., M. Remy-Zolghadri, D. Larouche, R. Gauvin, K. Baker, F. Bergeron, D. Dupuis, E. Langelier, D. Rancourt, F. A. Auger, and L. Germain. Tissue reorganization in response to mechanical load increases functionality. Tissue Eng. 11:90-100, 2005.

${ }^{53}$ Gruenbaum, Y., A. Margalit, R. D. Goldman, D. K. Shumaker, and K. L. Wilson. The nuclear lamina comes of age. Nat. Rev. Mol. Cell Biol. 6:21-31, 2005. 
${ }^{54}$ Gundersen, G. G., and H. J. Worman. Nuclear positioning. Cell 152:1376-1389, 2013.

${ }^{55}$ Guo, W. H., and Y. L. Wang. Retrograde fluxes of focal adhesion proteins in response to cell migration and mechanical signals. Mol. Biol. Cell 18:4519-4527, 2007.

${ }^{56}$ Haque, F., D. J. Lloyd, D. T. Smallwood, C. L. Dent, C. M. Shanahan, A. M. Fry, R. C. Trembath, and S. Shackleton. SUN1 interacts with nuclear lamin A and cytoplasmic nesprins to provide a physical connection between the nuclear lamina and the cytoskeleton. Mol. Cell Biol. 26:3738-3751, 2006.

${ }^{57}$ Harrison, R. G. The cultivation of tissues in extraneous media as a method of morphogenetic study. Anat. Rec. 6:181-193, 1912.

${ }^{58}$ Harvey, P. A., and L. A. Leinwand. The cell biology of disease: cellular mechanisms of cardiomyopathy. J. Cell Biol. 194:355-365, 2011.

${ }^{59}$ Hayakawa, K., A. Hosokawa, K. Yabusaki, and T. Obinata. Orientation of smooth muscle-derived A10 cells in culture by cyclic stretching: relationship between stress fiber rearrangement and cell reorientation. Zool. Sci. 17:617624, 2000.

${ }^{60}$ Hayakawa, K., N. Sato, and T. Obinata. Dynamic reorientation of cultured cells and stress fibers under mechanical stress from periodic stretching. Exp. Cell Res. 268:104-114, 2001.

${ }^{61}$ Hinton, Jr., R. B., J. Lincoln, G. H. Deutsch, H. Osinska, P. B. Manning, D. W. Benson, and K. E. Yutzey. Extracellular matrix remodeling and organization in developing and diseased aortic valves. Circ. Res. 98:1431-1438, 2006.

${ }^{62}$ Ho, C. Y., D. E. Jaalouk, M. K. Vartiainen, and J. Lammerding. Lamin A/C and emerin regulate MKL1-SRF activity by modulating actin dynamics. Nature 497:507-511, 2013.

${ }^{63}$ Ho, C. Y., and J. Lammerding. Lamins at a glance. J. Cell Sci. 125:2087-2093, 2012.

${ }^{64}$ Hotulainen, P., O. Llano, S. Smirnov, K. Tanhuanpaa, J. Faix, C. Rivera, and P. Lappalainen. Defining mechanisms of actin polymerization and depolymerization during dendritic spine morphogenesis. J. Cell Biol. 185:323-339, 2009.

${ }^{65}$ Houtchens, G. R., M. D. Foster, T. A. Desai, E. F. Morgan, and J. Y. Wong. Combined effects of microtopography and cyclic strain on vascular smooth muscle cell orientation. J. Biomech. 41:762-769, 2008.

${ }^{66} \mathrm{Hsu}$, H. J., C. F. Lee, and R. Kaunas. A dynamic stochastic model of frequency-dependent stress fiber alignment induced by cyclic stretch. PLoS One 4:e4853, 2009.

${ }^{67}$ Hsu, H. J., C. F. Lee, A. Locke, S. Q. Vanderzyl, and R. Kaunas. Stretch-induced stress fiber remodeling and the activations of JNK and ERK depend on mechanical strain rate, but not FAK. PLoS One 5:e12470, 2010.

${ }^{68}$ Humphries, M. J. Integrin structure. Biochem. Soc. Trans. 28:311-339, 2000.

${ }^{69}$ Ingber, D. E. Mechanobiology and diseases of mechanotransduction. Ann. Med. 35:564-577, 2003.

${ }^{70}$ Jungbauer, S., H. Gao, J. P. Spatz, and R. Kemkemer. Two characteristic regimes in frequency-dependent dynamic reorientation of fibroblasts on cyclically stretched substrates. Biophys. J. 95:3470-3478, 2008.

${ }^{71}$ Kakisis, J. D., E. D. Avgerinos, and C. D. Liapis. Analysis of the evidence behind the ESVS guidelines for the invasive treatment of carotid stenosis. Acta Chir. Belg. 109:574-580, 2009.

${ }^{72}$ Kaunas, R., and H. J. Hsu. A kinematic model of stretchinduced stress fiber turnover and reorientation. J. Theor. Biol. 257:320-330, 2009.
${ }^{73}$ Kaunas, R., P. Nguyen, S. Usami, and S. Chien. Cooperative effects of Rho and mechanical stretch on stress fiber organization. Proc. Natl. Acad. Sci. USA 102:15895-15900, 2005.

${ }^{74}$ Kaunas, R., S. Usami, and S. Chien. Regulation of stretchinduced JNK activation by stress fiber orientation. Cell Signal. 18:1924-1931, 2006.

${ }^{75}$ Khatau, S. B., C. M. Hale, P. J. Stewart-Hutchinson, M. S. Patel, C. L. Stewart, P. C. Searson, D. Hodzic, and D. Wirtz. A perinuclear actin cap regulates nuclear shape. Proc. Natl. Acad. Sci. USA 106:19017-19022, 2009.

${ }^{76}$ Khatau, S. B., D. H. Kim, C. M. Hale, R. J. Bloom, and D. Wirtz. The perinuclear actin cap in health and disease. Nucleus. 1:337-342, 2010.

${ }^{77}$ Kim, D. H., S. Cho, and D. Wirtz. Tight coupling between nucleus and cell migration through the perinuclear actin cap. J. Cell Sci. 127:2528-2541, 2014.

${ }^{78}$ Kim, D. H., and D. Wirtz. Cytoskeletal tension induces the polarized architecture of the nucleus. Biomaterials 48:161172, 2015.

${ }^{79}$ Kurpinski, K., J. Chu, C. Hashi, and S. Li. Anisotropic mechanosensing by mesenchymal stem cells. Proc. Natl. Acad. Sci. USA 103:16095-16100, 2006.

${ }^{80}$ Lammerding, J., L. G. Fong, J. Y. Ji, K. Reue, C. L. Stewart, S. G. Young, and R. T. Lee. Lamins A and C but not lamin B1 regulate nuclear mechanics. J. Biol. Chem. 281:25768-25780, 2006.

${ }^{81}$ Lammerding, J., P. C. Schulze, T. Takahashi, S. Kozlov, T. Sullivan, R. D. Kamm, C. L. Stewart, and R. T. Lee. Lamin A/C deficiency causes defective nuclear mechanics and mechanotransduction. J. Clin. Invest 113:370-378, 2004.

${ }^{82}$ Lee, C. F., C. Haase, S. Deguchi, and R. Kaunas. Cyclic stretch-induced stress fiber dynamics-dependence on strain rate, Rho-kinase and MLCK. Biochem. Biophys. Res. Commun. 401:344-349, 2010.

${ }^{83}$ Lee, J. S., C. M. Hale, P. Panorchan, S. B. Khatau, J. P. George, Y. Tseng, C. L. Stewart, D. Hodzic, and D. Wirtz. Nuclear lamin A/C deficiency induces defects in cell mechanics, polarization, and migration. Biophys. $J$. 93:2542-2552, 2007.

${ }^{84}$ Lele, T. P., J. Pendse, S. Kumar, M. Salanga, J. Karavitis, and D. E. Ingber. Mechanical forces alter zyxin unbinding kinetics within focal adhesions of living cells. J. Cell Physiol. 207:187-194, 2006.

${ }^{85}$ Li, P., P. Meinke, L. T. T. Huong, M. Wehnert, and A. A. Noegel. Contribution of SUN1 mutations to the pathomechanism in muscular dystrophies. Hum. Mutat. 35:452461, 2014.

${ }^{86}$ Li, R., Y. Wu, A. M. Manso, Y. Gu, P. Liao, S. Israeli, T. Yajima, U. Nguyen, M. S. Huang, N. D. Dalton, K. L. Peterson, and R. S. Ross. betal integrin gene excision in the adult murine cardiac myocyte causes defective mechanical and signaling responses. Am. J. Pathol. 180:952-962, 2012.

${ }^{87}$ Liang, W. C., H. Mitsuhashi, E. Keduka, I. Nonaka, S. Noguchi, I. Nishino, and Y. K. Hayashi. TMEM43 mutations in emery-dreifuss muscular dystrophy-related myopathy. Ann. Neurol. 69:1005-1013, 2011.

${ }^{88}$ Liu, B., M. J. Qu, K. R. Qin, H. Li, Z. K. Li, B. R. Shen, and Z. L. Jiang. Role of cyclic strain frequency in regulating the alignment of vascular smooth muscle cells in vitro. Biophys. J. 94:1497-1507, 2008.

${ }^{89}$ Livne, A., E. Bouchbinder, and B. Geiger. Cell reorientation under cyclic stretching. Nat. Commun. 5:3938, 2014.

${ }^{90}$ Lo, S. H. Focal adhesions: what's new inside. Dev. Biol. 294:280-291, 2006. 
${ }^{91}$ Lombardi, M. L., D. E. Jaalouk, C. M. Shanahan, B. Burke, K. J. Roux, and J. Lammerding. The interaction between nesprins and sun proteins at the nuclear envelope is critical for force transmission between the nucleus and cytoskeleton. J. Biol. Chem. 286:26743-26753, 2011.

${ }^{92}$ MacKenna, D., S. R. Summerour, and F. J. Villarreal. Role of mechanical factors in modulating cardiac fibroblast function and extracellular matrix synthesis. Cardiovasc. Res. 46:257-263, 2000.

${ }^{93}$ Maniotis, A. J., C. S. Chen, and D. E. Ingber. Demonstration of mechanical connections between integrins, cytoskeletal filaments, and nucleoplasm that stabilize nuclear structure. Proc. Natl. Acad. Sci. USA 94:849-854, 1997.

${ }^{94}$ Meinke, P., E. Mattioli, F. Haque, S. Antoku, M. Columbaro, K. R. Straatman, H. J. Worman, G. G. Gundersen, G. Lattanzi, M. Wehnert, and S. Shackleton. Muscular dystrophy-associated SUN1 and SUN2 variants disrupt nuclear-cytoskeletal connections and myonuclear organization. PLoS Genet. 10:e1004605, 2014.

${ }^{95}$ Mogilner, A., and G. Oster. Cell motility driven by actin polymerization. Biophys. J. 71:3030-3045, 1996.

${ }^{96}$ Muntoni, F., G. Bonne, L. G. Goldfarb, E. Mercuri, R. J. Piercy, M. Burke, R. Ben Yaou, P. Richard, D. Recan, A. Shatunov, C. A. Sewry, and S. C. Brown. Disease severity in dominant Emery Dreifuss is increased by mutations in both emerin and desmin proteins. Brain 129:1260-1268, 2006.

${ }^{97} \mathrm{Na}$, S., O. Collin, F. Chowdhury, B. Tay, M. Ouyang, Y. Wang, and N. Wang. Rapid signal transduction in living cells is a unique feature of mechanotransduction. Proc. Natl. Acad. Sci. USA 105:6626-6631, 2008.

${ }^{98}$ Nagayama, K., S. Yamazaki, Y. Yahiro, and T. Matsumoto. Estimation of the mechanical connection between apical stress fibers and the nucleus in vascular smooth muscle cells cultured on a substrate. J. Biomech. 47:14221429, 2014.

${ }^{99}$ Neidlinger-Wilke, C., E. Grood, L. Claes, and R. Brand. Fibroblast orientation to stretch begins within three hours. J. Orthop. Res. 20:953-956, 2002.

${ }^{100}$ Neidlinger-Wilke, C., E. S. Grood, J. H. C. Wang, R. A. Brand, and L. Claes. Cell alignment is induced by cyclic changes in cell length: studies of cells grown in cyclically stretched substrates. J. Orthop. Res. 19:286-293, 2001.

${ }^{101} \mathrm{Ngu}$, H., Y. Feng, L. Lu, S. J. Oswald, G. D. Longmore, and F. C. Yin. Effect of focal adhesion proteins on endothelial cell adhesion, motility and orientation response to cyclic strain. Ann. Biomed. Eng. 38:208-222, 2010.

${ }^{102}$ Niwa, K., J. K. Perloff, S. M. Bhuta, H. Laks, D. C. Drinkwater, J. S. Child, and P. D. Miner. Structural abnormalities of great arterial walls in congenital heart disease: light and electron microscopic analyses. Circulation 103:393-400, 2001.

${ }^{103}$ Nobes, C. D., and A. Hall. Rho GTPases control polarity, protrusion, and adhesion during cell movement. J. Cell Biol. 144:1235-1244, 1999.

${ }^{104}$ Oakes, P. W., and M. L. Gardel. Stressing the limits of focal adhesion mechanosensitivity. Curr. Opin. Cell Biol. 30:68-73, 2014.

${ }^{105}$ Oakley, C., and D. M. Brunette. The sequence of alignment of microtubules, focal contacts and actin filaments in fibroblasts spreading on smooth and grooved titanium substrata. J. Cell Sci. 106(Pt 1):343-354, 1993.

${ }^{106}$ Obbink-Huizer, C., C. W. Oomens, S. Loerakker, J. Foolen, C. V. Bouten, and F. P. Baaijens. Computational model predicts cell orientation in response to a range of mechanical stimuli. Biomech. Model. Mechanobiol. 13:227-236, 2014.

${ }^{107}$ Ohara, P. T., and R. C. Buck. Contact guidance in vitro. A light, transmission, and scanning electron microscopic study. Exp. Cell Res. 121:235-249, 1979.

${ }^{108}$ Padmakumar, V. C., T. Libotte, W. S. Lu, H. Zaim, S. Abraham', A. A. Noegel, J. Gotzmann, R. Foisner, and L. Karakesisoglou. The inner nuclear membrane protein Sun 1 mediates the anchorage of Nesprin-2 to the nuclear envelope. J. Cell Sci. 118:3419-3430, 2005.

${ }^{109}$ Pajerowski, J. D., K. N. Dahl, F. L. Zhong, P. J. Sammak, and D. E. Discher. Physical plasticity of the nucleus in stem cell differentiation. Proc. Natl. Acad. Sci. USA 104:15619-15624, 2007.

${ }^{110}$ Papadaki, M., N. Bursac, R. Langer, J. Merok, G. Vunjak-Novakovic, and L. E. Freed. Tissue engineering of functional cardiac muscle: molecular, structural, and electrophysiological studies. Am. J. Physiol Heart Circ. Physiol. 280:H168-H178, 2001.

${ }^{111}$ Poh, Y. C., S. P. Shevtsov, F. Chowdhury, D. C. Wu, S. $\mathrm{Na}, \mathrm{M}$. Dundr, and N. Wang. Dynamic force-induced direct dissociation of protein complexes in a nuclear body in living cells. Nat. Commun. 3:866, 2012.

${ }^{112}$ Prodanov, L., J. te Riet, E. Lamers, M. Domanski, R. Luttge, J. J. van Loon, J. A. Jansen, and X. F. Walboomers. The interaction between nanoscale surface features and mechanical loading and its effect on osteoblastlike cells behavior. Biomaterials 31:7758-7765, 2010.

${ }^{113}$ Puklin-Faucher, E., M. Gao, K. Schulten, and V. Vogel. How the headpiece hinge angle is opened: new insights into the dynamics of integrin activation. J. Cell Biol. 175:349-360, 2006

${ }^{114}$ Puklin-Faucher, E., and M. P. Sheetz. The mechanical integrin cycle. J. Cell Sci. 122:179-186, 2009.

${ }^{115}$ Saez, A., M. Ghibaudo, A. Buguin, P. Silberzan, and B. Ladoux. Rigidity-driven growth and migration of epithelial cells on microstructured anisotropic substrates. Proc. Natl. Acad. Sci. USA 104:8281-8286, 2007.

${ }^{116}$ Saito, A. C., T. S. Matsui, T. Ohishi, M. Sato, and S. Deguchi. Contact guidance of smooth muscle cells is associated with tension-mediated adhesion maturation. Exp. Cell Res. 327:1-11, 2014.

${ }^{117}$ Samarel, A. M. Focal adhesion signaling in heart failure. Pflugers Arch. 466:1101-1111, 2014.

${ }^{118}$ Sands, G., S. Goo, D. Gerneke, I. LeGrice, and D. Loiselle. The collagenous microstructure of cardiac ventricular trabeculae carneae. J. Struct. Biol. 173:110-116, 2011.

${ }^{119}$ Sarkar, S., M. Dadhania, P. Rourke, T. A. Desai, and J. Y. Wong. Vascular tissue engineering: microtextured scaffold templates to control organization of vascular smooth muscle cells and extracellular matrix. Acta Biomater. 1:93-100, 2005.

${ }^{120}$ Schoen, F. J. Aortic valve structure-function correlations: role of elastic fibers no longer a stretch of the imagination. J. Heart Valve Dis. 6:1-6, 1997.

${ }^{121}$ Schwarz, U. S., and M. L. Gardel. United we stand: integrating the actin cytoskeleton and cell-matrix adhesions in cellular mechanotransduction. J. Cell Sci. 125:3051-3060, 2012.

${ }^{122}$ Simon, D. N., and K. L. Wilson. The nucleoskeleton as a genome-associated dynamic 'network of networks'. Nat. Rev. Mol. Cell Biol. 12:695-708, 2011.

${ }^{123}$ Smith, M. A., E. Blankman, N. O. Deakin, L. M. Hoffman, C. C. Jensen, C. E. Turner, and M. C. Beckerle. LIM 
domains target actin regulators paxillin and zyxin to sites of stress fiber strain. PLoS One 8:e69378, 2013.

${ }^{124}$ Smith, M. A., L. M. Hoffman, and M. C. Beckerle. LIM proteins in actin cytoskeleton mechanoresponse. Trends Cell Biol. 24:575-583, 2014.

${ }^{125}$ Sosa, B. A., A. Rothballer, U. Kutay, and T. U. Schwartz. LINC complexes form by binding of three KASH peptides to domain interfaces of trimeric SUN proteins. Cell 149:1035-1047, 2012.

${ }^{126}$ Standley, P. R., A. Cammarata, B. P. Nolan, C. T. Purgason, and M. A. Stanley. Cyclic stretch induces vascular smooth muscle cell alignment via NO signaling. Am. J. Physiol Heart Circ. Physiol 283:H1907-H1914, 2002.

${ }^{127}$ Starr, D. A. KASH and SUN proteins. Curr. Biol. 21:R414-R415, 2011

${ }^{128}$ Tamiello, C., C. V. Bouten, and F. P. Baaijens. Competition between cap and basal actin fiber orientation in cells subjected to contact guidance and cyclic strain. Sci. Rep. 5:8752, 2015.

${ }^{129}$ Taranum, S., E. Vaylann, P. Meinke, S. Abraham, L. Yang, S. Neumann, L. Karakesisoglou, M. Wehnert, and A. A. Noegel. LINC complex alterations in DMD and EDMD/ CMT fibroblasts. Eur. J. Cell Biol. 91:614-628, 2012.

${ }^{130}$ Teixeira, A. I., G. A. Abrams, P. J. Bertics, C. J. Murphy, and P. F. Nealey. Epithelial contact guidance on well-defined micro- and nanostructured substrates. J. Cell Sci. 116:1881-1892, 2003.

${ }^{131}$ Tojkander, S., G. Gateva, and P. Lappalainen. Actin stress fibers - assembly, dynamics and biological roles. $J$. Cell Sci. 125:1855-1864, 2012.

${ }^{132}$ Tomasek, J. J., and E. D. Hay. Analysis of the role of microfilaments and microtubules in acquisition of bipolarity and elongation of fibroblasts in hydrated collagen gels. J. Cell Biol. 99:536-549, 1984.

${ }^{133}$ Tondon, A., H. J. Hsu, and R. Kaunas. Dependence of cyclic stretch-induced stress fiber reorientation on stretch waveform. J. Biomech. 45:728-735, 2012.

${ }^{134}$ Tranquillo, R. T., T. S. Girton, B. A. Bromberek, T. G. Triebes, and D. L. Mooradian. Magnetically orientated tissue-equivalent tubes: application to a circumferentially orientated media-equivalent. Biomaterials 17:349-357, 1996.

${ }^{135}$ Uttayarat, P., G. K. Toworfe, F. Dietrich, P. I. Lelkes, and R. J. Composto. Topographic guidance of endothelial cells on silicone surfaces with micro- to nanogrooves: orientation of actin filaments and focal adhesions. $J$. Biomed. Mater. Res. A 75:668-680, 2005.

${ }^{136}$ van Kooten, T. G., and A. F. von Recum. Cell adhesion to textured silicone surfaces: the influence of time of adhesion and texture on focal contact and fibronectin fibril formation. Tissue Eng. 5:223-240, 1999.

${ }^{137}$ Ventre, M., C. F. Natale, C. Rianna, and P. A. Netti. Topographic cell instructive patterns to control cell adhesion, polarization and migration. J. R. Soc. Interface 11:20140687, 2014.

${ }^{138}$ Versaevel, M., T. Grevesse, and S. Gabriele. Regulation of nuclear shape and function with cell elongation. Biophys. J. 104:151A, 2013.

${ }^{139}$ Vishavkarma, R., S. Raghavan, C. Kuyyamudi, A. Majumder, J. Dhawan, and P. A. Pullarkat. Role of actin filaments in correlating nuclear shape and cell spreading. PLoS One 9:e107895, 2014.

${ }^{140}$ Vogel, V., and M. Sheetz. Local force and geometry sensing regulate cell functions. Nat. Rev. Mol. Cell Biol. $7: 265-275,2006$
${ }^{141}$ Walboomers, X. F., L. A. Ginsel, and J. A. Jansen. Early spreading events of fibroblasts on microgrooved substrates. J. Biomed. Mater. Res. 51:529-534, 2000.

${ }^{142}$ Walboomers, X. F., W. Monaghan, A. S. Curtis, and J. A. Jansen. Attachment of fibroblasts on smooth and microgrooved polystyrene. J. Biomed. Mater. Res. 46:212-220, 1999.

${ }^{143}$ Wang, J. H. Substrate deformation determines actin cytoskeleton reorganization: a mathematical modeling and experimental study. J. Theor. Biol. 202:33-41, 2000.

${ }^{144}$ Wang, J. H., P. Goldschmidt-Clermont, J. Wille, and F. C. Yin. Specificity of endothelial cell reorientation in response to cyclic mechanical stretching. J. Biomech. 34:1563-1572, 2001.

${ }^{145}$ Wang, J. H., P. Goldschmidt-Clermont, and F. C. Yin. Contractility affects stress fiber remodeling and reorientation of endothelial cells subjected to cyclic mechanical stretching. Ann. Biomed. Eng. 28:1165-1171, 2000.

${ }^{146}$ Wang, J. H., and E. S. Grood. The strain magnitude and contact guidance determine orientation response of fibroblasts to cyclic substrate strains. Connect. Tissue Res. 41:29-36, 2000.

${ }^{147}$ Wang, J. H., E. S. Grood, J. Florer, and R. Wenstrup. Alignment and proliferation of MC3T3-E1 osteoblasts in microgrooved silicone substrata subjected to cyclic stretching. J. Biomech. 33:729-735, 2000.

${ }^{148}$ Wang, H., W. Ip, R. Boissy, and E. S. Grood. Cell orientation response to cyclically deformed substrates: experimental validation of a cell model. J. Biomech. 28:1543-1552, 1995.

${ }^{149}$ Wang, N., J. D. Tytell, and D. E. Ingber. Mechanotransduction at a distance: mechanically coupling the extracellular matrix with the nucleus. Nat. Rev. Mol. Cell Biol. 10:75-82, 2009.

${ }^{150}$ Wang, D., Y. Xie, B. Yuan, J. Xu, P. Gong, and X. Jiang. A stretching device for imaging real-time molecular dynamics of live cells adhering to elastic membranes on inverted microscopes during the entire process of the stretch. Integr. Biol. (Camb.) 2:288-293, 2010.

${ }^{151}$ Wang, J. H., G. Yang, Z. Li, and W. Shen. Fibroblast responses to cyclic mechanical stretching depend on cell orientation to the stretching direction. J. Biomech. 37:573576,2004

${ }^{152}$ Wei, Z., V. S. Deshpande, R. M. McMeeking, and A. G. Evans. Analysis and interpretation of stress fiber organization in cells subject to cyclic stretch. J. Biomech. Eng. 130:031009, 2008.

${ }^{153}$ Wei, S. C., L. Fattet, J. H. Tsai, Y. Guo, V. H. Pai, H. E. Majeski, A. C. Chen, R. L. Sah, S. S. Taylor, A. J. Engler, and J. Yang. Matrix stiffness drives epithelial-mesenchymal transition and tumour metastasis through a TWIST1G3BP2 mechanotransduction pathway. Nat. Cell Biol. 17:678-688, 2015.

${ }^{154}$ Weichsel, J., N. Herold, M. J. Lehmann, H. G. Krausslich, and U. S. Schwarz. A quantitative measure for alterations in the actin cytoskeleton investigated with automated high-throughput microscopy. Cytom. Part A 77A:52-63, 2010.

${ }^{155}$ Weiss, P. Experiments on cell and axon orientation in vitro; the role of colloidal exudates in tissue organization. J. Exp. Zool. 100:353-386, 1945.

${ }^{156}$ Whitehead, J., D. Vignjevic, C. Futterer, E. Beaurepaire, S. Robine, and E. Farge. Mechanical factors activate betacatenin-dependent oncogene expression in APC mouse colon. HFSP. J. 2:286-294, 2008. 
${ }^{157}$ Wille, J. J., C. M. Ambrosi, and F. C. Yin. Comparison of the effects of cyclic stretching and compression on endothelial cell morphological responses. J. Biomech. Eng. 126:545-551, 2004.

${ }^{158}$ Williams, C., A. W. Xie, M. Yamato, T. Okano, and J. Y. Wong. Stacking of aligned cell sheets for layer-by-layer control of complex tissue structure. Biomaterials 32:56255632, 2011.

${ }^{159}$ Worman, H. J. Nuclear lamins and laminopathies. $J$. Pathol. 226:316-325, 2012.

${ }^{160}$ Yamane, M., T. Matsuda, T. Ito, Y. Fujio, K. Takahashi, and J. Azuma. Racl activity is required for cardiac myocyte alignment in response to mechanical stress. Biochem. Biophys. Res. Commun. 353:1023-1027, 2007.

${ }^{161}$ Yoshigi, M., E. B. Clark, and H. J. Yost. Quantification of stretch-induced cytoskeletal remodeling in vascular endothelial cells by image processing. Cytom. A 55:109-118, 2003.

${ }^{162}$ Yoshigi, M., L. M. Hoffman, C. C. Jensen, H. J. Yost, and M. C. Beckerle. Mechanical force mobilizes zyxin from focal adhesions to actin filaments and regulates cytoskeletal reinforcement. J. Cell Biol. 171:209-215, 2005.

${ }^{163}$ Zaidel-Bar, R., C. Ballestrem, Z. Kam, and B. Geiger. Early molecular events in the assembly of matrix adhesions at the leading edge of migrating cells. J. Cell Sci. 116:4605-4613, 2003.

${ }^{164}$ Zhang, Q. P., C. Bethmann, N. F. Worth, J. D. Davies, C. Wasner, A. Feuer, C. D. Ragnauth, Q. J. Yi, J. A. Mellad,
D. T. Warren, M. A. Wheeler, J. A. Ellis, J. N. Skepper, M. Vorgerd, B. Schlotter-Weigel, P. L. Weissberg, R. G. Roberts, M. Wehnert, and C. M. Shanahan. Nesprin-1 and -2 are involved in the pathogenesis of Emery-Dreifuss muscular dystrophy and are critical for nuclear envelope integrity. Hum. Mol. Genet. 16:2816-2833, 2007.

${ }^{165}$ Zhang, L., C. J. Kahn, H. Q. Chen, N. Tran, and X. Wang. Effect of uniaxial stretching on rat bone mesenchymal stem cell: orientation and expressions of collagen types I and III and tenascin-C. Cell Biol. Int. 32:344-352, 2008.

${ }^{166}$ Zhong, Z., K. L. Wilson, and K. N. Dahl. Beyond lamins other structural components of the nucleoskeleton. Methods Cell Biol. 98:97-119, 2010.

${ }^{167}$ Zimerman, B., M. Arnold, J. Ulmer, J. Blummel, A. Besser, J. P. Spatz, and B. Geiger. Formation of focal adhesion-stress fibre complexes coordinated by adhesive and non-adhesive surface domains. IEE. Proc. Nanobiotechnol. 151:62-66, 2004.

${ }^{168}$ Zuela, N., D. Z. Bar, and Y. Gruenbaum. Lamins in development, tissue maintenance and stress. Embo Rep. 13:1070-1078, 2012.

${ }^{169}$ Zwerger, M., D. E. Jaalouk, M. L. Lombardi, P. Isermann, M. Mauermann, G. Dialynas, H. Herrmann, L. L. Wallrath, and J. Lammerding. Myopathic lamin mutations impair nuclear stability in cells and tissue and disrupt nucleo-cytoskeletal coupling. Hum. Mol. Genet. 22:23352349, 2013. 